\title{
Comments on Janocha et al. Lie Symmetry Analysis of the Hopf Functional-Differential Equation. Symmetry 2015, 7, 1536-1566
}

\author{
Michael Frewer ${ }^{1, *}$ and George Khujadze ${ }^{2}$ \\ 1 Trübnerstraße 42, 69121 Heidelberg, Germany \\ 2 Chair of Fluid Mechanics, Universität Siegen, 57068 Siegen, Germany; george.khujadze@uni-siegen.de \\ * Correspondence: frewer.science@gmail.com \\ Academic Editor: Sergei Odintsov \\ Received: 3 October 2015; Accepted: 18 February 2016; Published: 18 April 2016
}

\begin{abstract}
The recent systematic study by Janocha et al. [1] to determine all possible Lie-point symmetries for the functional Hopf-Burgers equation is re-examined. From a more consistent theoretical framework, however, some of the proposed symmetry transformations of the considered Hopf-Burgers equation are in fact rejected. Three out of eight proposed symmetry transformations are invalidated, while two of them should be replaced by their correct intermediate formulations, but which ultimately violate internal consistency constraints of the governing equation. It is concluded that the recently proposed symmetry analysis method for functional integro-differential equations should not be adopted when aiming at a consistent and complete approach.
\end{abstract}

Keywords: Lie groups; Lie symmetries; Hopf equation; Burgers equation; functional-differential equations; symmetry breaking; turbulence

\section{Introduction: The Problem of Combining Implicit and Explicit Functional Dependence}

Next to the two different and well-established approaches of Ibragimov et al. [2,3] and of Fushchich and Zawistowski et al. [4-6] to systematically determine Lie symmetries of integro-differential equations, the latest study of Janocha et al. [1] claims to have found a third approach in how to perform a Lie-group symmetry analysis for such equations, in particular as to how this approach can be applied to integro-differential equations of the functional type (a general introduction into the theory of Lie-group symmetry analysis can be found, e.g., in [7-9]). It is claimed that this new, third approach allows for a standard Lie-point symmetry analysis without having to directly transform the volume element of integration. Listed as the third item in [1] (p. 1549), this approach is intended to avoid the "more complicated" [1] (p. 1549) approaches of Ibragimov et al. (which rests on a Lie-Bäcklund analysis) and that of Fushchich and Zawistowski et al. (which incorporates the transformation of the volume element (Jacobian) within a standard Lie-point analysis), when specifically extended to functional equations in the sense as described in [1].

However, as we will show in this section, this third approach, as it is used throughout in [1] to systematically determine all Lie-point symmetries of the functional Hopf-Burgers equation in physical space, is based on a wrong fundamental assumption and several subsequent reasoning errors. (Remark: Note that via the Cole-Hopf transformation, $c f$. [10], the general solution of the deterministic Burgers and, thus, also of the statistical Hopf-Burgers equation can be formally expressed in closed form; attempts to evaluate these solutions can be found, e.g., in [11,12]). The consequence for the final result in [1]: the determined symmetries $X_{4}^{\text {phys }}-X_{6}^{\text {phys }}$ listed in Table 2 [1] (p. 1562) are, in actual fact, not symmetries, i.e., these Lie-group transformations are not admitted as symmetries by the considered 
functional Hopf-Burgers equation (the proof is given in Appendix A). Before we reveal all errors of this approach in [1], it is necessary to first clarify two essential points:

(i) Caution has to be exercised when using the notation of Hopf [13] for the functional derivative (shown here only for the 1D case):

$$
\frac{\delta}{\delta y(x)}=\frac{\partial}{\partial y(x) d x}
$$

The right-hand notation was introduced by Hopf only for the sole reason to explicitly show the dimensional character of the functional derivative, in that it carries the physical dimension:

$$
\left[\frac{\delta}{\delta y(x)}\right]=\frac{1}{[y] \cdot L}
$$

where $[y]$ is the physical dimension of the variable $y$ and $L$ the dimension of length. This notation, however, should not be misused, e.g., by interpreting the symbol " $y(x) d x$ " in Equation (1) as a usual product between the variables $y$ and $d x$, with the aim to identify it then as a stand-alone new independent variable $\omega:=y(x) \cdot d x$. In fact, the only active variable in Equation (1) is $y$. Its argument, however, i.e., the variable $x$, is a so-called silent variable. That means, the independent variable $x$ is not actively participating in the process of a functional variation; it only acts passively in Equation (1) as a bookkeeping variable in the sense of a continuous index.

(ii) When considering a transformation, e.g., on the 2D set of variables $\left(y\left(x^{\prime}\right), x\right) \mapsto\left(\bar{y}\left(\bar{x}^{\prime}\right), \bar{x}\right)$, it is necessary to realize that there is only one physical space, i.e., only one set $G \subseteq \mathbb{R}^{1}$ where both spatial variables $x^{\prime}$ and $x$ belong to: $x^{\prime}, x \in G$. Hence, when treating $x^{\prime}$ and $x$ as true variables, i.e., as quantities that can vary between all values in $G$, the transformation rule for $x^{\prime} \mapsto \bar{x}^{\prime}$ must obviously be the same as for $x \mapsto \bar{x}$. Consequently, the transformation on the above set of variables can also be formally written as $(y(x), x) \mapsto(\bar{y}(\bar{x}), \bar{x})$. To simplify formal expressions, we will make use of this notation, as long as no ambiguity arises.

Having these two points in mind, let us now look more closely at the notation, definitions and assumptions in [1], which should form the basis of a new approach towards determining all Lie-point symmetries of the (spatially unbounded) functional Hopf-Burgers equation in physical space:

$$
\frac{\partial \Phi}{\partial t}=\int y(x)\left(i \frac{\partial}{\partial x} \frac{\delta^{2} \Phi}{\delta y(x) \delta y(x)}+v \frac{\partial^{2}}{\partial x^{2}} \frac{\delta \Phi}{\delta y(x)}\right) d x
$$

which is presented in [1] as Equation [8] (here and below, in square brackets, we denote equations from [1]; also note that for the considered Lie-group symmetry analysis, the integration domain $G$ in Equation [8] was later chosen for the sake of simplicity as unbounded $G=(-\infty,+\infty)$ [1] (p. 1551)). When working with Equation (3), three things should be pointed out here:

(a) In order to perform a consistent Lie-group symmetry analysis for a functional differential equation (FDE), such as Equation (3), it is crucial to correctly identify and separate the independent from the dependent variables. Looking at Equation (3), it is clear that $t$ and $x$ are to be identified as independent variables and $\Phi$ as a dependent one. But, how does one identify the variable $y=y(x)$ ? Is it a dependent or an independent variable? Therefore, in order to arrive at unique results, we must specify the order of the mathematical operations in Equation (3). Since by construction the functional derivatives have priority over the usual differentiation and integration processes (note that, due to this priority of the functional derivatives over the partial derivatives, they do not commute, that means $\frac{\partial}{\partial x} \frac{\delta}{\delta y(x)} \neq \frac{\delta}{\delta y(x)} \frac{\partial}{\partial x}$ ), the variable $y$ has to be identified as an independent variable, which additionally can be differentiated relative to $x$, i.e., $\partial_{x} y=y^{\prime}(x)$. This property of having different dependencies of $y$, namely the hierarchy of being an independent functional variable relative to $x$, which then can be differentiated to it, has to be carefully monitored in Equation (3) when transforming this equation, otherwise one runs into a conflict of 
dependencies. But, this exact monitoring has not been done in [1], and thus, a conflict of variables takes place throughout that study.

(b) The dependent variable $\Phi$ in Equation (3) only depends on the functional variable $y$ and on time $t$ :

$$
\Phi=\Phi(y(x), t)
$$

It does not explicitly depend on the variable $x$, i.e., we always yield the evaluation $\partial_{x} \Phi=0$. Note that in the characteristic functional $\Phi$, we do not make use of the notation in representing the dependency on $y$ by additional square brackets, as used throughout in [1]. It is redundant information, which unnecessarily overloads the notation. Suppressing these square brackets still will keep the notation unique. Hence, to allow for a more readable notation, we will change to it in the following.

(c) When $y$ acts as a dependent variable, it only depends on $x$, since the function $y=y(x)$ is defined as a time-independent function, i.e., $\partial_{t} y(x)=0$ (for more details, see [13]). Any symmetries thus found must be compatible with this condition, i.e., in the transformed domain, we must obtain this independency, as well: $\partial_{\bar{t}} \bar{y}(\bar{x})=0$.

In [1], the construction of the Lie-point symmetry method for functional equations in physical space begins with a more general equation than the intended Hopf-Burgers equation. At first, a generic spatially 1D scalar FDE of third order is considered in Definition 5 [1] (p. 1542):

$$
F(y(x), x, t, \Phi, \underset{1}{\Phi}, \underset{2}{\Phi}, \underset{3}{\Phi})=0
$$

which has to undergo a Lie-group variable transformation. The independent variables are $y, x$ and $t$, while the only dependent variable is the functional $\Phi$, which, at first, should show an explicit dependence on all involved independent variables, i.e., $\Phi=\Phi(y(x), x, t)$, which then later in Definition 6 [1] (p. 1544) is reduced to the explicit $x$-independent Hopf functional $\Phi=\Phi(y(x), t)$. Now, when considering the most general set of Lie-point symmetries that Equation (5) can admit, it will have the structure:

$$
\left.\begin{array}{rl}
\bar{y} & =y+\xi_{y}(y, x, t, \Phi) \varepsilon \\
\bar{x} & =x+\xi_{x}(y, x, t, \Phi) \varepsilon \\
\bar{t} & =t+\xi_{t}(y, x, t, \Phi) \varepsilon \\
\bar{\Phi} & =\Phi+\eta_{\Phi}(y, x, t, \Phi) \varepsilon
\end{array}\right\}
$$

where $\varepsilon$ is the group parameter and where the $\xi^{\prime}$ 's and $\eta$ are the infinitesimals of the corresponding independent and dependent variable transformations, respectively. But, instead of using the transparent structure of Equation (6), the authors in [1] introduce a new independent variable, the combined (differential) variable:

$$
\omega:=y \cdot d x
$$

which during the transformation process should replace the single variable $y$. It forms the core idea of the new approach to "transform $y(z) d z$ instead of $y(z)$ " [1] (p. 1549), with the assumed argument to have the advantage of circumventing the transformation of the volume element $d x$, which, since it only will come in the combined form of Equation (7), can be absorbed into the variable $y$. Hence, instead of Equation (6), the following Lie-group structure for Equation (5) is considered in Definition 5 [1] (p. 1542):

$$
\left.\begin{array}{rl}
\bar{\omega} & =\omega+\xi_{\omega}(y, x, t, \Phi) \varepsilon \\
\bar{x} & =x+\xi_{x}(y, x, t, \Phi) \varepsilon \\
\bar{t} & =t+\xi_{t}(y, x, t, \Phi) \varepsilon \\
\bar{\Phi} & =\Phi+\eta_{\Phi}(y, x, t, \Phi) \varepsilon
\end{array}\right\}
$$


where the infinitesimal $\xi_{\omega}$ is denoted as the misleading symbol " $\xi_{y} d x^{\prime \prime}$ [1] (p. 1542), since it suggests the relationship $\xi_{\omega}=\xi_{y} \cdot d x$, which, as we will show further below, is not valid. Note that the dependency of $\xi_{\omega}$ in Definition 5 [1] (p. 1542) is formally extended by a further spatial variable $z$, but which, when referring to the above Discussion (ii), can be regarded as redundant information, which can be safely suppressed. Two fundamental problems immediately arise when using this new structure of Equation (8) instead of the classical Lie-point transformation structure of Equation (6):

(1) From the information that one directly gets from the new approach of Equation (8), it is unclear how the functional variable $y$ should transform. The only way to retrieve this information is to enforce the consistent condition $\bar{\omega}=\bar{y} \cdot d \bar{x}$, which will lead to:

$$
\begin{aligned}
\omega+\xi_{\omega} \varepsilon=\bar{\omega}=\overline{y \cdot d x} & =\bar{y} \cdot d \bar{x} \\
& =\left(y+\xi_{y} \varepsilon\right) \cdot\left(d x+\partial_{x} \xi_{x} d x \varepsilon\right) \\
& =y \cdot d x+\left(\xi_{y} d x+y \cdot \partial_{x} \xi_{x} d x\right) \varepsilon+\mathcal{O}\left(\varepsilon^{2}\right)
\end{aligned}
$$

from which, then, the following relationship between $\xi_{\omega}, \xi_{y}$ and $\xi_{x}$ can be read off:

$$
\xi_{\omega}=\left(\xi_{y}+y \cdot \partial_{x} \xi_{x}\right) d x
$$

(Remark: Note that identifying $\bar{\omega}$ as the combined transformed variable $\bar{\omega}=\bar{y} \cdot d \bar{x}$ is also practiced in [1], e.g., in Equation [11] and Equations [12]-[14]; the term $d \bar{x}$ denotes here the transformed $1 \mathrm{D}$ volume element (Jacobian) and not the total variation of $\bar{x}$ relative to the variables $y, x, t$ and $\Phi$, as it is set up in Equation (8)). Given thus the transformation for $\omega$ and $x$, the transformation for $y$ is then retrieved by solving the above Equation (10) for its infinitesimal $\xi_{y}$. Note again that the term $J:=1+\varepsilon \partial_{x} \xi_{x}$ represents the 1D Jacobian for the infinitesimal transformation of the 1D variable $x$. Compared to the above consistent result for $\xi_{\omega}$ of Equation (10), we now see that the notation $\xi_{\omega}=\xi_{y} d x$ as used in [1] (pp. 1542-1543) is more than misleading, since it only reflects at most half of the information given by Equation (10).

(2) The more severe problem, which essentially turns the proposed symmetry analysis in [1] into an incomplete and also inconsistent analysis, is the fact that the dependent variable $\Phi$, as defined in Definition 5 [1] (p. 1542), only depends on $y, x$ and $t$ and not on the combined variable $\omega$ of Equation (7). Surely, instead of the original system of Equation (5), one can consider a more general system in rewriting the original system $F$ equivalently into:

$$
F\left(\omega, y, x, t, \Phi^{*}, \underset{1}{\Phi^{*}}, \underset{2}{\Phi^{*}}, \Phi_{3}^{*}\right)=0
$$

by allowing at the same time for a more general functional $\Phi^{*}$, which then includes the dependency of $\omega$ as an additional independent variable: $\Phi^{*}=\Phi^{*}(\omega, y, x, t)$. Such an extended system will of course admit at first a more general set of symmetries than the original system when consistently looking for invariant transformations of the form:

$$
\left.\begin{array}{rl}
\bar{\omega} & =\omega+\xi_{\omega}\left(\omega, y, x, t, \Phi^{*}\right) \varepsilon \\
\bar{y} & =y+\xi_{y}\left(\omega, y, x, t, \Phi^{*}\right) \varepsilon \\
\bar{x} & =x+\xi_{x}\left(\omega, y, x, t, \Phi^{*}\right) \varepsilon \\
\bar{t} & =t+\xi_{t}\left(\omega, y, x, t, \Phi^{*}\right) \varepsilon \\
\bar{\Phi}^{*} & =\Phi^{*}+\eta_{\Phi^{*}}\left(\omega, y, x, t, \Phi^{*}\right) \varepsilon
\end{array}\right\}
$$

but when putting the consistency condition $\omega=y \cdot d x$ of Equation (7), along with its transformational restriction $\xi_{\omega}=\left(\xi_{y}+y \cdot \partial_{x} \xi_{x}\right) d x$ of Equation (10), back into the obtained generalized result of Equation (12), it will consistently break the set of symmetries down to the admitted set of the original Equation (5). But, such a consistently extended analysis has not been 
performed in [1], which forms the key element of our critique: instead of performing (along with the condition $\omega=y \cdot d x$ ) the complete and consistent analysis of Equation (12) for a system as Equation (11), the incomplete and inconsistent analysis of Equation (8) for system Equation (5) is performed, with the consequence that the determined symmetries $X_{4}^{\text {phys }}-X_{6}^{\text {phys }}$ listed in [1] (p. 1562) are in fact not admitted as symmetries of the considered system (see Appendix A). In addition, it is not clear whether then the remaining list of Lie-point symmetries is also complete. It is very likely that essential symmetries cannot be captured by such an approach as proposed in [1]; indications for this supposition are given by several examples of possible Hopf-functionals in Equations (15)-(17) further below, which cannot be solely expressed by $\omega$ within a system of the form of Equation (5).

To close this section, let us briefly illustrate the latter critique (2) at the concrete example of the functional Hopf-Burgers Equation (3) as it is considered in [1] from Definition 6 [1] (p. 1544) onwards. As it was just discussed before, in order to perform a consistent symmetry analysis when involving the additional independent (differential) variable $\omega=\omega(d x)$ of Equation (7), one can consider for the Hopf-Burgers Equation (3), instead of the original system of Equation (5), the generalized system of Equation (11), which then takes the form:

$$
\frac{\partial \Phi^{*}\left(\omega\left(d x^{\prime}\right), y\left(x^{\prime}\right), t\right)}{\partial t}=\int \omega(d x)\left(i \frac{\partial}{\partial x} \frac{\delta^{2} \Phi^{*}\left(\omega\left(d x^{\prime}\right), y\left(x^{\prime}\right), t\right)}{\delta y(x) \delta y(x)}+v \frac{\partial^{2}}{\partial x^{2}} \frac{\delta \Phi^{*}\left(\omega\left(d x^{\prime}\right), y\left(x^{\prime}\right), t\right)}{\delta y(x)}\right)
$$

When determining the Lie-point symmetries of this system in the structural form of Equation (12), one then obtains an extended set of symmetries, but which will systematically reduce to the set of symmetries admitted by the original system of Equation (3) once the consistency condition $\omega(d x)=y \cdot d x$ of Equation (7), along with its transformational restriction $\xi_{\omega}=\left(\xi_{y}+y \cdot \partial_{x} \xi_{x}\right) d x$ of Equation (10), is enforced upon it. (Remark: Note that this approach can be straightforwardly realized in 1D. However, for functional equations operating in a higher dimensional physical space, like the 3D Hopf-Navier-Stokes equation, for example, it is questionable whether this approach, which involves more variables than the original system, is of any real advantage when compared to the other two existing and well-established approaches of Ibragimov, Fushchich and Zawistowksi et al. In particular, since the aim of this third approach, namely to avoid the transformation of the volume element (Jacobian), is never really accomplished; it will always come back through the consistency relation of Equation (10)). But, instead of Equation (13), the following extended system is analyzed in [1]:

$$
\frac{\partial \Phi\left(y\left(x^{\prime}\right), t\right)}{\partial t}=\int \omega(d x)\left(i \frac{\partial}{\partial x} \frac{\partial^{2} \Phi\left(y\left(x^{\prime}\right), t\right)}{\partial \omega(d x) \partial \omega(d x)}+v \frac{\partial^{2}}{\partial x^{2}} \frac{\partial \Phi\left(y\left(x^{\prime}\right), t\right)}{\partial \omega(d x)}\right)
$$

to determine its Lie-point symmetries in the reduced form of Equation (8), which thus consequently constitutes an incomplete and inconsistent analysis. As was just discussed before in a more general setting, the problem is that the Hopf-functional $\Phi$ only depends on $y$ and $t$ and not necessarily on the combined variable structure $\omega(d x)=y \cdot d x$ of Equation (7). Here are three distinct and well-defined counter-examples:

$$
\begin{aligned}
& \Phi_{1}(y, t)=\exp \left(i f_{1}(t) \int d x^{\prime} \Theta\left(y\left(x^{\prime}\right)\right) y\left(x^{\prime}\right)^{2}\right), \quad f_{1}(t) \neq 0, \Theta(y(x))=y(x) /|y(x)| \\
& \Phi_{2}(y, t)=\exp \left(i \int d x^{\prime} d x^{\prime \prime} f_{2}\left(x^{\prime \prime}\right) y\left(x^{\prime \prime}\right) y\left(x^{\prime}\right) y\left(x^{\prime}+c\right)\right), \quad f_{2}(x) \neq 0, c \neq 0 \\
& \Phi_{3}(y, t)=\exp \left(-\left(\int d x^{\prime} f_{3}\left(x^{\prime}, t\right) y\left(x^{\prime}\right) y\left(x^{\prime}+x\right)\right)^{2}\right), \quad y(0) \neq 0, f_{3}(0, t) \neq 0
\end{aligned}
$$

where $f_{1}, f_{2}$ and $f_{3}$ are arbitrary real-valued functions with their constraints as given above and where $\Theta(x)=x /|x|$ is the antisymmetric Heaviside function. (Remark: It should be clear that the 
examples of Equations (15)-(17) do not present particular solutions of the Hopf-Burgers Equation (3); instead, they only should serve as examples to show which solution structures of the Hopf-Burgers equation are possible). All three examples cannot be solely expressed through the variable $\omega=y \cdot d x$, despite the fact that they all represent realizable Hopf-functionals, in not only satisfying the underlying natural (physical) constraints (see Definition 11 [1] (p. 1559)), but also having well-conditioned first and second order functional derivatives, such that the integral kernel in the Hopf-Burgers Equation (3) is well-defined. For example, when considering the functional Equation (15), we yield the two well-defined kernel terms:

$$
\begin{gathered}
i y(x) \frac{\partial}{\partial x} \frac{\delta^{2} \Phi_{1}}{\delta y(x) \delta y(x)}=-8 i y^{\prime}(x)\left(f_{1}(t) \Theta(y(x)) y(x)\right)^{2} \exp \left(i f_{1}(t) \int d x^{\prime} \Theta\left(y\left(x^{\prime}\right)\right) y\left(x^{\prime}\right)^{2}\right) \\
v y(x) \frac{\partial^{2}}{\partial x^{2}} \frac{\delta \Phi_{1}}{\delta y(x)}=2 i v y^{\prime \prime}(x) f_{1}(t) \Theta(y(x)) y(x) \exp \left(i f_{1}(t) \int d x^{\prime} \Theta\left(y\left(x^{\prime}\right)\right) y\left(x^{\prime}\right)^{2}\right)
\end{gathered}
$$

(Remark: In contrast, the two specifications $\Phi(y, t)=\exp \left(i y\left(x^{\prime}\right)\right)$ or $\Phi(y, t)=\exp \left(i \int d x^{\prime} y\left(x^{\prime}\right)^{3}\right)$, do not constitute realizable Hopf-functionals, for example, since their second functional derivatives $\frac{\delta^{2} \Phi}{\delta y(x) \delta y(x)}$ are ill-conditioned, in that they give rise to a squared Dirac delta function, which cannot be removed inside the integral kernel of Equation (3)).

Hence, the striking difference between the consistent extension of Equation (13) for the Hopf-Burgers Equation (3) and its inconsistent one, Equation (14), as analyzed in [1], is that the former formulation of Equation (13) still correctly defines the functional derivatives relative to the original variable $y$ and not to $\omega$, which would be incorrect.

\section{A Proposal for a Consistent Treatment in Generating Lie-Point Symmetries for FDEs}

In this section, we develop a complete and consistent approach to systematically find all possible Lie-point symmetries for FDEs, without having to claim that it represents an alternative or novel approach. It is just based on the classical Lie-point symmetry method for differential equations extended by the well-established ideas of Fushchich and Zawistowski et al. for integro-differential equations [4-6], when prolonged to also include functional variations. No further extensions or generalizations of any kind, for example as we put forward in Equation (11), are needed. This correct ansatz should then replace the inconsistent one in [1]. (Remark: First attempts to extend the classical Lie-point symmetry analysis from partial differential and integro-differential to functional equations can be found in [14,15]; a shortcoming of these attempts is discussed in [16]).

The technical aim of this section is to show how the correct prolonged infinitesimals of the original Hopf-Burgers Equation (3) can be constructed. We will explicitly demonstrate this method only for the first order prolonged infinitesimals $\zeta_{; t}$ and $\zeta_{; y(x)}$, which then should be used instead of the incorrect results presented in [1]. The construction of all other prolonged infinitesimals is then carried out analogously. However, before we present this method, it will be helpful to briefly repeat the key idea of how to correctly construct a prolonged infinitesimal in terms of the given or wanted point infinitesimals for a usual partial differential equation (PDE), in order to then transfer it to FDEs.

\subsection{Construction Guideline for Prolongations in the Example of a PDE}

Let us demonstrate the construction principle in the example of the 1D diffusion equation:

$$
\frac{\partial u(t, x)}{\partial t}=v \frac{\partial^{2} u(t, x)}{\partial x^{2}}
$$

To find the infinitesimals $\zeta_{; t}$ and $\zeta_{; x}$ (in the notation of [1]) for the transformed first order differential variables $\bar{u}_{, \bar{t}}:=\frac{\partial \bar{u}}{\partial \bar{t}}$ and $\bar{u}_{, \bar{x}}:=\frac{\partial \bar{u}}{\partial \bar{x}}$ in their consistent form:

$$
\bar{u}_{, \bar{t}}=u, t+\zeta_{; t}(t, x, u, u, t, u, x) \varepsilon, \quad \bar{u}_{, \bar{x}}=u_{, x}+\zeta_{; x}(t, x, u, u, t, u, x) \varepsilon
$$


when considering the following Lie-group of point transformations in the infinitesimal form:

$$
\bar{t}=t+\xi_{t}(t, x, u) \varepsilon, \quad \bar{x}=x+\xi_{x}(t, x, u) \varepsilon, \quad \bar{u}=u+\eta(t, x, u) \varepsilon
$$

one has to determine the total variation of the dependent variable $u$ relative to the independent variables $t$ and $x$, which in the transformed domain is given as:

$$
d \bar{u}(\bar{t}, \bar{x})=\bar{u}_{, \bar{t}} d \bar{t}+\bar{u}_{, \bar{x}} d \bar{x}
$$

Because, according to Equation (22), this total variation can also be evaluated as:

$$
\begin{aligned}
d \bar{u}(\bar{t}, \bar{x}) & =d u(t, x)+\varepsilon \cdot d \eta(t, x, u) \\
& =u_{, t} d t+u_{, x} d x+\varepsilon \cdot\left(\eta, t d t+\eta, x d x+\eta, u\left(u_{, t} d t+u_{, x} d x\right)\right)
\end{aligned}
$$

which, when taking the total variations of the infinitesimally-transformed independent variables of Equation (22):

$$
\begin{aligned}
d \bar{t} & =d t+\varepsilon \cdot d \xi_{t}(t, x, u) \\
& =d t+\varepsilon \cdot\left(\xi_{t, t} d t+\xi_{t, x} d x+\xi_{t, u}\left(u_{, t} d t+u_{, x} d x\right)\right) \\
d \bar{x} & =d x+\varepsilon \cdot d \xi_{x}(t, x, u) \\
& =d x+\varepsilon \cdot\left(\xi_{x, t} d t+\xi_{x, x} d x+\xi_{x, u}\left(u_{, t} d t+u_{, x} d x\right)\right)
\end{aligned}
$$

and inverting these for the untransformed total variations $d t$ and $d x$ (in terms of the transformed ones) by collecting only terms up to first order in the group parameter $\varepsilon$, to get:

$$
\begin{aligned}
& d t=d \bar{t}-\varepsilon \cdot\left(\xi_{t, t} d \bar{t}+\xi_{t, x} d \bar{x}+\xi_{t, u}\left(u_{t, t} d \bar{t}+u_{, x} d \bar{x}\right)\right) \\
& d x=d \bar{x}-\varepsilon \cdot\left(\xi_{x, t} d \bar{t}+\xi_{x, x} d \bar{x}+\xi_{x, u}\left(u_{, t} d \bar{t}+u_{, x} d \bar{x}\right)\right)
\end{aligned}
$$

in order to insert these back into Equation (24), one then finally can read off (up to first order in $\varepsilon$ ) the transformation structure of both first order differential variables $\bar{u}_{,}, \bar{t}$ and $\bar{u}_{,} \bar{x}$ when comparing them to Equation (23). The well-known result for Equation (21) is thus given by:

$$
\zeta_{; t}=\frac{\mathcal{D} \eta}{\mathcal{D} t}-u_{, t} \frac{\mathcal{D} \xi_{t}}{\mathcal{D} t}-u_{, x} \frac{\mathcal{D} \xi_{x}}{\mathcal{D} t}, \quad \zeta_{; x}=\frac{\mathcal{D} \eta}{\mathcal{D} x}-u_{, t} \frac{\mathcal{D} \xi_{t}}{\mathcal{D} x}-u_{, x} \frac{\mathcal{D} \xi_{x}}{\mathcal{D} x}
$$

where $\mathcal{D} / \mathcal{D} t$ and $\mathcal{D} / \mathcal{D} x$ are the total variation operators for the independent variables $t$ and $x$, respectively, whose explicit expansion forms depend on the corresponding variable dependence of the functions they act on, i.e., here, for the dependencies as given by Equation (22), they take the explicit form:

$$
\frac{\mathcal{D}}{\mathcal{D} t}=\frac{\partial}{\partial t}+u, t \frac{\partial}{\partial u}, \quad \frac{\mathcal{D}}{\mathcal{D} x}=\frac{\partial}{\partial x}+u, x \frac{\partial}{\partial u}
$$

\subsection{First Order Prolongations for the Functional Hopf-Burgers Equation}

The construction principle developed above can now be straightforwardly applied to FDEs, as, e.g., to the Hopf-Burgers Equation (3). Given are three independent variables $t, x$ and $y$ and one dependent variable $\Phi$, which does not explicitly depend on $x$ (see Discussions ( $a$ ) and (b) in Section 1). 
Due to this explicit non-dependency on $x$, the corresponding differential variable is zero in both the untransformed, as well as in the transformed domain:

$$
\Phi_{, x}:=\frac{\partial \Phi}{\partial x}=0, \quad \bar{\Phi}_{, \bar{x}}:=\frac{\partial \bar{\Phi}}{\partial \bar{x}}=0
$$

Hence, the aim is only to find the infinitesimals $\zeta_{; t}$ and $\zeta_{; y(x)}$ for the two remaining transformed first order differential variables $\bar{\Phi}_{, \bar{t}}:=\frac{\partial \bar{\Phi}}{\partial \bar{t}}$ and $\bar{\Phi}_{, \bar{y}(\bar{x})}:=\frac{\delta \bar{\Phi}}{\delta \bar{y}(\bar{x})}$ in their consistent form:

$$
\bar{\Phi}_{, \bar{t}}=\Phi_{, t}+\zeta_{; t}\left(t, x, y(x), \Phi, \Phi_{, t}, \Phi_{, y(x)}\right) \varepsilon, \quad \bar{\Phi}_{, \bar{y}(\bar{x})}=\Phi_{, y(x)}+\zeta_{; y(x)}\left(t, x, y(x), \Phi, \Phi_{, t}, \Phi_{, y(x)}\right) \varepsilon
$$

when considering the following Lie-group of point transformations in the infinitesimal form:

$$
\left.\begin{array}{rl}
\bar{t} & =t+\xi_{t}(t, x, y(x), \Phi) \varepsilon \\
\bar{x} & =x+\xi_{x}(t, x, y(x), \Phi) \varepsilon \\
\bar{y}(\bar{x}) & =y(x)+\xi_{y(x)}(t, x, y(x), \Phi) \varepsilon \\
\bar{\Phi} & =\Phi+\eta(t, x, y(x), \Phi) \varepsilon
\end{array}\right\}
$$

(Remark: Note that at this stage, we will consider all infinitesimals in Equation (33) first in their most general form. Their consistency with the constraints $\bar{\Phi}_{, \bar{x}}=0$ and $\bar{y}_{, \bar{t}}=0$ will be investigated later. Further note that in [1], the infinitesimal for the single dependent variable $\Phi$ is denoted as $\eta_{\Phi}$. In this study, however, we prefer to denote it just as $\eta$ ).

The total (functional) variation of $\Phi$ relative to the independent variables $t, x$ and $y$ is given in the transformed domain as:

$$
\delta \bar{\Phi}(\bar{t}, \bar{y}(\bar{x}))=\bar{\Phi}_{, \bar{t}} d \bar{t}+\int d \bar{x}^{\prime} \bar{\Phi}_{, \bar{y}\left(\bar{x}^{\prime}\right)} \delta \bar{y}\left(\bar{x}^{\prime}\right)
$$

which, according to Equation (33), can also be evaluated as:

$$
\begin{aligned}
\delta \bar{\Phi}(\bar{t}, \bar{y}(\bar{x}))= & \delta \Phi(t, y(x))+\varepsilon \cdot \delta \eta(t, x, y(x), \Phi) \\
= & \Phi_{, t} d t+\int d x^{\prime} \Phi_{, y\left(x^{\prime}\right)} \delta y\left(x^{\prime}\right) \\
& \quad+\varepsilon \cdot\left(\eta_{, t} d t+\eta_{, x} d x+\int d x^{\prime} \eta_{, y\left(x^{\prime}\right)} \delta y\left(x^{\prime}\right)+\eta_{, \Phi}\left(\Phi_{, t} d t+\int d x^{\prime} \Phi_{, y\left(x^{\prime}\right)} \delta y\left(x^{\prime}\right)\right)\right)
\end{aligned}
$$

Analogously, the evaluation for the total (functional) variation of the infinitesimally-transformed independent variables of Equation (33), which are given as:

$$
\begin{aligned}
d \bar{t} & =d t+\varepsilon \cdot \delta \xi_{t}(t, x, y(x), \Phi) \\
& =d t+\varepsilon \cdot\left(\xi_{t, t} d t+\xi_{t, x} d x+\int d x^{\prime} \xi_{t, y\left(x^{\prime}\right)} \delta y\left(x^{\prime}\right)+\xi_{t, \Phi} \delta \Phi\right) \\
d \bar{x} & =d x+\varepsilon \cdot \delta \xi_{x}(t, x, y(x), \Phi) \\
& =d x+\varepsilon \cdot\left(\xi_{x, t} d t+\xi_{x, x} d x+\int d x^{\prime} \xi_{x, y\left(x^{\prime}\right)} \delta y\left(x^{\prime}\right)+\xi_{x, \Phi} \delta \Phi\right) \\
\delta \bar{y}(\bar{x}) & =\delta y(x)+\varepsilon \cdot \delta \xi_{y(x)}(t, x, y(x), \Phi) \\
& =\delta y(x)+\varepsilon \cdot\left(\xi_{y(x), t} d t+\xi_{y(x), x} d x+\int d x^{\prime} \xi_{y(x), y\left(x^{\prime}\right)} \delta y\left(x^{\prime}\right)+\xi_{y(x), \Phi} \delta \Phi\right)
\end{aligned}
$$


with $\delta \Phi=\Phi_{, t} d t+\int d x^{\prime} \Phi_{, y\left(x^{\prime}\right)} \delta y\left(x^{\prime}\right)$. When identifying the above transformations in the form of:

$$
\left.\begin{array}{rl}
d \bar{t} & =d t+\varepsilon \cdot \mathcal{F}_{t}(d t, d x, \delta y(x)) \\
d \bar{x} & =d x+\varepsilon \cdot \mathcal{F}_{x}(d t, d x, \delta y(x)) \\
\delta \bar{y}(\bar{x}) & =\delta y(x)+\varepsilon \cdot \mathcal{F}_{y(x)}(d t, d x, \delta y(x))
\end{array}\right\}
$$

where the functionals $\mathcal{F}_{\alpha_{i}}$, with the placeholders $\alpha_{1}=t, \alpha_{2}=x$ and $\alpha_{3}=y(x)$, are defined as:

$$
\begin{aligned}
\mathcal{F}_{\alpha_{i}}\left(d \alpha_{1}, d \alpha_{2}, \delta \alpha_{3}\right)=\xi_{\alpha_{i}, t} d \alpha_{1}+\xi_{\alpha_{i}, x} d \alpha_{2}+\int d x^{\prime} \xi_{\alpha_{i}, y\left(x^{\prime}\right)} & \delta \alpha_{3}^{\prime} \\
& +\xi_{\alpha_{i}, \Phi}\left(\Phi_{, t} d \alpha_{1}+\int d x^{\prime} \Phi_{, y\left(x^{\prime}\right)} \delta \alpha_{3}^{\prime}\right)
\end{aligned}
$$

then the inverse transformations of Equations (36)-(38) (up to first order in $\varepsilon$ ) take the form:

$$
\left.\begin{array}{rl}
d t & =d \bar{t}-\varepsilon \cdot \mathcal{F}_{t}(d \bar{t}, d \bar{x}, \delta \bar{y}(\bar{x})) \\
d x & =d \bar{x}-\varepsilon \cdot \mathcal{F}_{x}(d \bar{t}, d \bar{x}, \delta \bar{y}(\bar{x})) \\
\delta y(x) & =\delta \bar{y}(\bar{x})-\varepsilon \cdot \mathcal{F}_{y(x)}(d \bar{t}, d \bar{x}, \delta \bar{y}(\bar{x}))
\end{array}\right\}
$$

since the functionals $\mathcal{F}_{\alpha_{i}}$ of Equation (40), can only undergo a change of zeroth order to yield in each case a first order transformation of the corresponding variables. Indeed, substituting Equation (41) back into Equation (39) will reduce each equation to an identity transformation up to first order:

$$
d \bar{t}=d \bar{t}+\mathcal{O}\left(\varepsilon^{2}\right), \quad d \bar{x}=d \bar{x}+\mathcal{O}\left(\varepsilon^{2}\right), \quad \delta \bar{y}(\bar{x})=\delta \bar{y}(\bar{x})+\mathcal{O}\left(\varepsilon^{2}\right)
$$

Now, when inserting the inverse transformations of Equation (41) into $\delta \bar{\Phi}$ of Equation (35) and matching it to its form of Equation (34), then one obtains the following transformation rule for the first order differential variables of Equation (32):

$$
\begin{gathered}
\bar{\Phi}_{, \bar{t}}=\Phi_{, t}+\varepsilon \cdot\left(\eta_{, t}+\Phi_{, t}\left(\eta, \Phi-\xi_{t, t}\right)-\Phi_{, t}^{2} \xi_{t, \Phi}\right. \\
\left.-\int d x^{\prime} \Phi_{, y\left(x^{\prime}\right)} \xi_{y\left(x^{\prime}\right), t}-\int d x^{\prime} \Phi_{, y\left(x^{\prime}\right)} \Phi_{, t} \xi_{y\left(x^{\prime}\right), \Phi}\right) \\
d \bar{x}^{\prime} \bar{\Phi}_{, \bar{y}\left(\bar{x}^{\prime}\right)}=d x^{\prime} \Phi_{, y\left(x^{\prime}\right)}+\varepsilon \cdot d x^{\prime} \cdot\left(\eta_{, y\left(x^{\prime}\right)}+\Phi_{, y\left(x^{\prime}\right)} \eta_{, \Phi}-\Phi_{, t} \xi_{t, y\left(x^{\prime}\right)}-\Phi_{, t} \Phi_{, y\left(x^{\prime}\right)} \xi_{t, \Phi}\right. \\
\left.-\int d x^{\prime \prime} \Phi_{, y\left(x^{\prime \prime}\right)} \xi_{y\left(x^{\prime \prime}\right), y\left(x^{\prime}\right)}-\int d x^{\prime \prime} \Phi_{, y\left(x^{\prime \prime}\right)} \Phi_{, y\left(x^{\prime}\right)} \xi_{y\left(x^{\prime \prime}\right), \Phi}\right)
\end{gathered}
$$

along with the constraint equation:

$$
\eta_{, x}-\Phi_{, t} \xi_{t, x}-\int d x^{\prime} \Phi_{, y\left(x^{\prime}\right)} \xi_{y\left(x^{\prime}\right), x}=0
$$

which arises due to the zero term $\bar{\Phi}_{, \bar{x}} d \bar{x}=0$ not explicitly appearing in the underlying total variation of Equation (34). Hence, we automatically gained with this process the restricting Equation (45) on the generally assumed form of the infinitesimals in Equation (33), such that they are all compatible with the underlying restriction $\Phi_{, x}=0$ of the Hopf functional $\Phi$. Note that we also could have derived Equation (45) in a different way, by just rewriting the constraint $\bar{\Phi}_{, \bar{x}}=\partial_{\bar{x}} \bar{\Phi}=0$ into its 
expanded form using the transformation rules of Equation (33) and their inverses up to first order in the group parameter:

$$
\begin{aligned}
0 & =\partial_{\bar{x}} \bar{\Phi}=\left(\frac{\partial t}{\partial \bar{x}} \partial_{t}+\frac{\partial x}{\partial \bar{x}} \partial_{x}+\int d x^{\prime} \frac{\partial y\left(x^{\prime}\right)}{\partial \bar{x}} \frac{\delta}{\delta y\left(x^{\prime}\right)}\right)(\Phi+\varepsilon \cdot \eta)+\mathcal{O}\left(\varepsilon^{2}\right) \\
& =\frac{\partial t}{\partial \bar{x}} \partial_{t} \Phi+\int d x^{\prime} \frac{\partial y\left(x^{\prime}\right)}{\partial \bar{x}} \frac{\delta \Phi}{\delta y\left(x^{\prime}\right)}+\varepsilon \cdot\left(\frac{\partial t}{\partial \bar{x}} \partial_{t} \eta+\frac{\partial x}{\partial \bar{x}} \partial_{x} \eta+\int d x^{\prime} \frac{\partial y\left(x^{\prime}\right)}{\partial \bar{x}} \frac{\delta \eta}{\delta y\left(x^{\prime}\right)}\right)+\mathcal{O}\left(\varepsilon^{2}\right) \\
& =-\varepsilon \frac{\partial \xi_{t}}{\partial \bar{x}} \partial_{t} \Phi-\varepsilon \int d x^{\prime} \frac{\partial \xi_{y\left(x^{\prime}\right)}}{\partial \bar{x}} \frac{\delta \Phi}{\delta y\left(x^{\prime}\right)}+\varepsilon \partial_{x} \eta+\mathcal{O}\left(\varepsilon^{2}\right) \\
& =\left(\partial_{x} \eta-\frac{\partial \xi_{t}}{\partial x} \partial_{t} \Phi-\int d x^{\prime} \frac{\partial \xi_{y\left(x^{\prime}\right)}}{\partial x} \frac{\delta \Phi}{\delta y\left(x^{\prime}\right)}\right) \varepsilon+\mathcal{O}\left(\varepsilon^{2}\right)
\end{aligned}
$$

which then results in Equation (45). (Remark: Note that in the third to last line in Equation (46), we have used the fact that the partial derivatives $\xi_{t, \bar{x}}$ and $\xi_{y\left(x^{\prime}\right), \bar{x}}$ can only undergo a change of zeroth order, i.e., $\xi_{t, \bar{x}}=\xi_{t, x}$ and $\xi_{y\left(x^{\prime}\right), \bar{x}}=\xi_{y\left(x^{\prime}\right), x}$, in order to yield an overall transformation up to first order in the group parameter $\varepsilon$ ). Now, when comparing all of the above results with those obtained in [1], we notice the following remarkable differences:

D.1: Equation [18] in [1] and the subsequent equation below it, each contain two terms in the prolonged infinitesimals for the correspondingly-transformed first order variables $\bar{\Phi}_{, \bar{t}}$ and $\bar{\Phi}_{, y\left(z_{1}\right)}[1]$ (p. 1543), namely the last two terms in $\zeta_{; t}$ and $\zeta_{; y(x)}$ [1] (p. 1546), which are not present in our corresponding results of Equations (43) and (44). These terms being proportional to the variations of the infinitesimal $\xi_{x}$ appear to be due to a technical error performed in [1], which we will discuss in detail in the next section (see Discussions E.1 and E.2)

$D .2$ : The prolonged infinitesimal $\zeta_{; y(x)}$ resulting from Equation (44) also differs in another, independent respect from the result obtained in [1] (p. 1546). To see this difference, one first has to recognize that with Equation (44), we did not directly obtain a transformation rule for the differential variable $\Phi_{, y(x)}$; instead, we only obtained a rule for the combined product variable $\Phi_{, y(x)} d x$. To retrieve the transformation rule just for the variable $\Phi_{, y(x)}$, we first have to transform the 1D volume element $d x$, which for the infinitesimal transformation $x \mapsto \bar{x}$ of Equation (33) up to first order is expressed through the 1D Jacobian:

$$
d x=\left(1-\varepsilon \cdot \xi_{x, x}\right) d \bar{x}+\mathcal{O}\left(\varepsilon^{2}\right)
$$

which then allows us to formulate the transformation rule for $\Phi_{, y(x)}$ as:

$$
\bar{\Phi}_{, \bar{y}(\bar{x})}=\Phi_{, y(x)}+\varepsilon \cdot \zeta_{; y(x)}+\mathcal{O}\left(\varepsilon^{2}\right)
$$

with the corresponding prolonged infinitesimal:

$$
\begin{aligned}
\zeta_{; y(x)}=-\Phi_{, y(x)} \xi_{x, x}+\eta_{, y(x)}+\Phi_{, y(x)} \eta_{, \Phi}-\Phi_{, t} \xi_{t, y(x)}-\Phi_{, t} \Phi_{, y(x)} \xi_{t, \Phi} & \\
& -\int d x^{\prime} \Phi_{, y\left(x^{\prime}\right)} \xi_{y\left(x^{\prime}\right), y(x)}-\int d x^{\prime} \Phi_{, y\left(x^{\prime}\right)} \Phi_{, y(x)} \xi_{y\left(x^{\prime}\right), \Phi}
\end{aligned}
$$

As we can see, the first term in the above result arising from the transformation of the volume element is not part of the derived result for $\zeta_{; y(x)}$ in [1] (p. 1546). The reason for this failure in [1] can be found in the discussions of the previous Section 1 (see in particular Discussion (2)) and certainly will continue to unfold for all higher order prolongations. Note that the approach done here to incorporate the transformation rule for the volume element into the overall transformation process is in accord with the well-established method of Fushchich and Zawistowski et al. [4-6] 
for integro-differential equations and, thus, does not constitute a novel approach here. In order to validate the result of Equation (49), let us consider, for example, the following simple scaling transformation in the point variables as given by $X_{1}^{\text {phys }}$ in Table 2 in [1] (p. 1562):

$$
\bar{t}=e^{2 \varepsilon} t=t+2 t \varepsilon+\mathcal{O}\left(\varepsilon^{2}\right), \quad \bar{x}=e^{\varepsilon} x=x+x \varepsilon+\mathcal{O}\left(\varepsilon^{2}\right), \quad \bar{y}(\bar{x})=y(x), \quad \bar{\Phi}=\Phi
$$

for which then the differential variable $\Phi_{, y(x)}$ must transform as:

$$
\bar{\Phi}_{, \bar{y}(\bar{x})}=e^{-\varepsilon} \Phi_{, y(x)}=\Phi_{, y(x)}-\varepsilon \Phi_{, y(x)}+\mathcal{O}\left(\varepsilon^{2}\right)
$$

simply because it carries the physical dimension (see Equation (2)):

$$
[\Phi, y(x)]=\left[\frac{\delta \Phi}{\delta y(x)}\right]=\frac{[\Phi]}{[y] \cdot L}
$$

which shows a scaling of $1 / x$, since the characteristic Hopf-functional $\Phi$ is dimensionless and $y$ transforms invariantly. Plugging the infinitesimals for the point transformations of Equation (50):

$$
\xi_{t}=2 t, \quad \xi_{x}=x, \quad \xi_{y(x)}=0, \quad \eta=0
$$

into the relation of Equation (49), we get the correct result for the prolonged infinitesimal of the first order differential variable $\Phi_{, y(x)}$, namely as it is given by Equation (51):

$$
\zeta_{; y(x)}=-\Phi, y(x)
$$

while when plugging it into the corresponding derived expression in [1] (p. 1546), we get the wrong result $\zeta_{; y(x)}=0$, when interpreting the combined infinitesimal $\xi_{y(x)} d x$ in the way that the underlying notation in [1] misleadingly suggests (e.g., as it is particularly denoted in Definition 5 along with Equation [11]), namely as a product of $\xi_{y(x)}$ and $d x$.

D.3: The restricting Equation (45) for the generally assumed infinitesimals of the point variables of Equation (33) automatically emerges as a consistent by-product in our analysis when systematically determining their first order prolongations under the restriction $\Phi_{, x}=0$. This result which, of course, reduces to the set of restrictions:

$$
\eta_{, x}=0, \quad \xi_{t, x}=0, \quad \xi_{y(x), x}=0
$$

is (or are) independent of the specific form of the underlying dynamical equation to be actually considered, i.e., the result of Equation (45) or its reduction to Equation (55) was obtained without providing any structural information from the Hopf-Burgers Equation (3) itself, except, of course, for the only information that its solution function must be explicitly independent of the spatial variable; in clear contrast to the derived restrictions of Equations [37] and [38] in [1] (p. 1554), which therein result only as solutions of the overdetermined system, which again has its explicit structure from the underlying equation to be considered. In particular, by closer inspection, we even observe the striking difference that the third restriction $\xi_{y(x), x}=0$ in Equation (55) does not coincide with Equation [37] in [1].

The difference is that all three restrictions in Equation (55) tell us that in order to warrant consistency with $\Phi_{, x}=0$, the infinitesimals $\eta, \xi_{t}$ and $\xi_{y(x)}$ should not show any explicit dependence on the spatial variable $x$, i.e., when looking in particular at $\xi_{y(x)}$, then, instead of the general form given in Equation (33), it should only show the reduced dependence $\xi_{y(x)}=\xi_{y(x)}(t, y(x), \Phi)$. However, Equation [37] in [1] does not induce this restriction, as can be readily seen, e.g., in the second term of Equation [47], which is treated as a non-zero term. Equation [37], therefore, does not induce the same restriction on the infinitesimal $\xi_{y(x)}$ as the 
corresponding and consistent restriction derived by us in Equation (55).

The problem is that the authors in [1] artificially distinguish between the terms $\xi_{y(z), x}$ and $\xi_{y(x), x}$ as given in Equations [37] and [47], respectively, if $z \neq x$. The reason is that in Definition 5 [1] (p. 1542), the infinitesimal $\xi_{y(x)}$ is misleadingly defined as a multipoint function depending explicitly on two spatial points $z$ and $x$. However, such a definition is inappropriate and, as we have shown in this study, is not even required in order to perform a consistent analysis (see also Discussion (ii) in Section 1). Hence, due to this multipoint definition in Definition 5 [1] (p. 1542), we face in Equation [37] the misleading consequence that $\xi_{y(z)}$ must be explicitly independent of the spatial variable $x$ while still depending explicitly on the spatial variable $z$. This can be clearly seen, e.g., in the proposed ansatz function for $\xi_{y(z)}$ in Equation [50], which is not compatible to the consistent constraint $\xi_{y(z), z}=0$ in Equation (55) when written relative to $z$.

Regarding the first restriction $\eta_{, x}=0$ in Equation (55), it is not clear from the analysis in [1] whether this consistent restriction has been initially assumed or not. Only in the end of their analysis, when solving the overdetermined system, a consistent ansatz function in Equation [45] is proposed in an ad hoc manner.

Finally, to close this section, we know that in order to perform a fully-consistent analysis, one also has to consider the second consistency condition $\partial_{t} y(x)=0$ (see Discussion (c) in Section 1). In order to determine possible restrictions for the infinitesimals, in the same sense as it was done for the condition $\partial_{x} \Phi=0$ in Equation (46), it is necessary to evaluate this condition $\partial_{t} y(x)=0$ according to Equations (33) and (55), which in the transformed domain up to first order then reads:

$$
\begin{aligned}
0 & =\partial_{\bar{t}} \bar{y}(\bar{x})=\left(\frac{\partial t}{\partial \bar{t}} \partial_{t}+\frac{\partial x}{\partial \bar{t}} \partial_{x}\right)\left(y(x)+\varepsilon \xi_{y(x)}\right)+\mathcal{O}\left(\varepsilon^{2}\right) \\
& =\frac{\partial x}{\partial \bar{t}} \partial_{x} y(x)+\varepsilon \cdot\left(\frac{\partial t}{\partial \bar{t}} \partial_{t} \xi_{y(x)}+\frac{\partial x}{\partial \bar{t}} \partial_{x} \xi_{y(x)}\right)+\mathcal{O}\left(\varepsilon^{2}\right)=-\varepsilon \frac{\partial \xi_{x}}{\partial \bar{t}} \partial_{x} y(x)+\varepsilon \partial_{t} \xi_{y(x)}+\mathcal{O}\left(\varepsilon^{2}\right) \\
& =\left(\xi_{x, t} \cdot y^{\prime}(x)-\xi_{y(x), t}\right) \varepsilon+\mathcal{O}\left(\varepsilon^{2}\right)
\end{aligned}
$$

This constraint equation can only be satisfied for all $y \neq 0$, if $\xi_{x, t}=0$ and $\xi_{y(x), t}=0$, i.e., if the infinitesimals for the variables $x$ and $y$ do not explicitly depend on time $t$. In other words, the infinitesimals as given through Equations (33) and (55) are only compatible with the constraint $\partial_{t} y(x)=0$ if $\xi_{x}$ and $\xi_{y(x)}$ are further restricted to be independent of $t$. From the final result given in Theorem 9 in [1] (p. 1558), it is clear that the above constraint Equation (56) has not been considered in their analysis, since the group parameters $a_{4}$ and $a_{5}$ are treated as non-zero quantities, but which, according to constraint Equation (56), must be zero; otherwise, we obtain symmetries that are not compatible with all internal consistency constraints of the Hopf-Burgers Equation (3).

\section{Points for Correction in [1]}

E.1: As written in [1], the variation of the transformed Hopf functional $\bar{\Phi}$ with respect to $t$ given through Equation [12] is incorrect. Their argument of why the temporal variation in the last term of Equation [12] is only acting on the transformed expansion coefficients $\bar{y}_{n}$ and not also on the orthogonal functions $h_{n}$ and the volume element $d \bar{x}^{\prime}$ is not convincing. Because, when following their preceding argument in decomposing $y(z) d z$ into a complete and transformable set of orthogonal basis functions, the correct variation up to first order will not lead to Equation [12], but instead, it will lead to:

$$
\frac{\mathcal{D} \bar{\Phi}}{\mathcal{D} t}=\frac{\mathcal{D} \bar{\Phi}}{\mathcal{D} \bar{t}} \frac{\mathcal{D} \overline{\mathcal{D}} t}{\mathcal{D} t}+\frac{\mathcal{D} \bar{\Phi}}{\mathcal{D} \bar{x}} \frac{\mathcal{D} \bar{x}}{\mathcal{D} t}+\int_{\bar{G}} \frac{\mathcal{D} \bar{\Phi}}{\mathcal{D} \overline{y\left(x^{\prime}\right) d x^{\prime}}} \sum_{n=1}^{\infty} \frac{\mathcal{D} \bar{y}_{n} h_{n}\left(\bar{x}^{\prime}-\varepsilon \xi_{x^{\prime}}\right) d \bar{x}^{\prime}}{\mathcal{D} t}
$$


This considerably influences all further results, e.g., the final result in Equation [15] is not given by three, but only by the first two terms on its right-hand side, which then coincides again with the above (correct) variation of Equation (57), knowing that $\mathcal{D} \bar{\Phi} / \mathcal{D} \bar{x}=0$. Hereby, it should be pointed out that the formulation for the transformed decomposition of $y(z) d z$ in Equation [11] is misleading, since it suggests that the basis functions transform frame-indifferently, i.e., as $h_{n}(z)=h_{n}(\bar{z})$, but which is not the case, as can be easily seen when directly transforming the initial decomposition for $y(z) d z$ in Equation [10] according to Definition 5 [1] (p. 1542) up to first order:

$$
\begin{aligned}
0 & =y(z) d z-\sum_{n=1}^{\infty} y_{n} h_{n}(z) d z=\overline{y(z) d z}-\varepsilon \xi_{y(z)} d z-\sum_{n=1}^{\infty} y_{n} h_{n}(z) d z+\mathcal{O}\left(\varepsilon^{2}\right) \\
& =\overline{y(z) d z}-\varepsilon \int_{G} d z^{\prime} \delta\left(z^{\prime}-z\right) \xi_{y\left(z^{\prime}\right)} d z-\sum_{n=1}^{\infty} y_{n} h_{n}(z) d z+\mathcal{O}\left(\varepsilon^{2}\right) \\
& =\overline{y(z) d z}-\varepsilon \int_{G} d z^{\prime} \xi_{y\left(z^{\prime}\right)} d z \sum_{n=1}^{\infty} h_{n}(z) h_{n}\left(z^{\prime}\right)-\sum_{n=1}^{\infty} y_{n} h_{n}(z) d z+\mathcal{O}\left(\varepsilon^{2}\right) \\
& =\overline{y(z) d z}-\sum_{n=1}^{\infty}\left(y_{n}+\varepsilon \int_{G} d z^{\prime} \xi_{y\left(z^{\prime}\right)} h_{n}\left(z^{\prime}\right)\right) h_{n}(z) d z+\mathcal{O}\left(\varepsilon^{2}\right) \\
& =\overline{y(z) d z}-\sum_{n=1}^{\infty}\left(y_{n}+\varepsilon \int_{G} d z^{\prime} \xi_{y\left(z^{\prime}\right)} h_{n}\left(z^{\prime}\right)\right) h_{n}(z)\left(1-\varepsilon \partial_{\bar{z}} \xi_{z}\right) d \bar{z}+\mathcal{O}\left(\varepsilon^{2}\right) \\
& =\overline{y(z) d z}-\sum_{n=1}^{\infty} \bar{y}_{n} \bar{h}_{n}(\bar{z}) d \bar{z}+\mathcal{O}\left(\varepsilon^{2}\right)
\end{aligned}
$$

where the transformation rules for $\bar{y}_{n}$ and $\bar{h}_{n}(\bar{z})$ are thus given as:

$$
\begin{gathered}
\bar{h}_{n}(\bar{z})=h_{n}(z)=h_{n}\left(\bar{z}-\varepsilon \xi_{z}\right) \neq h_{n}(\bar{z}) \\
\bar{y}_{n}=y_{n}+\varepsilon\left(\int_{G} d z^{\prime} \xi_{y\left(z^{\prime}\right)} h_{n}\left(z^{\prime}\right)-y_{n} \partial_{z} \xi_{z}\right)
\end{gathered}
$$

(Remark: We could not find any reasonable argument or see any analytical advantage for why the authors in [1] have made use of the formal expansion of Equation (58) at this stage of their analysis. In the way as their cited book by Klauder [17] already discusses in Section 3, in particular in Sections 3.5 and 3.7, this expansion only becomes relevant as soon as one specifies any functional for which one faces problems in practically evaluating its functional derivatives, where it sometimes is necessary to soften or to smear out the Dirac delta function. But, since all functionals in Section 2 of [1] are treated arbitrarily and no specifications are needed, such an expansion only unnecessarily complicates their analysis at this stage. Also, note that in the derivation of Equation (58) we assume that the set of basis functions $\left\{h_{n}(z)\right\}$ is complete and orthonormal, i.e., that they satisfy the relation $\left.\sum_{n=1}^{\infty} h_{n}\left(z^{\prime}\right) h_{n}(z)=\delta\left(z^{\prime}-z\right)\right)$.

Comparing now the above result of Equation (58) to Equation [11] up to first order, we see that the basis functions do not transform as $h(z)=h(\bar{z})$, as claimed in [1] (p. 1543), but instead as $h(z)=\bar{h}(\bar{z})$ as in Equation (59). Moreover, the expansion coefficients $\bar{y}_{n}$ do not transform independently, but are induced by the transformations of the variables $y(z) d z$ and $z$ according to Equation (60). However, note that the transformation of Equation (60) is not complete. It still must be supplemented by a consistency constraint, because when decomposing the time-independent 1D scalar (differential) variable $y(z) d z$ into a set of orthogonal functions $\left\{h_{n}(z)\right\}$ :

$$
y(z) d z=\sum_{n=1}^{\infty} y_{n} h_{n}(z) d z
$$


any variable transformation must of course be compatible with the following constraint, namely that the expansion coefficients $y_{n}$ constitute simple constants, i.e., $\partial_{t} y_{n}=\partial_{x} y_{n}=0$, which, if the set $\left\{h_{n}(z)\right\}$ is complete and orthonormal, are disclosed by:

$$
y_{n}=\int_{G} h_{n}(z) y(z) d z
$$

Hence, when transforming the relation of Equation (62), it will inherently induce restrictions on the transformation rule for the expansion coefficients $y_{n} \mapsto \bar{y}_{n}$ of Equation (60) and, thus, on the infinitesimals $\xi_{y(z)} d z$ and $\xi_{z}$. However, again, this information was not revealed in [1].

E.2: In going from Equation [13] to Equation [14], the authors in [1] assumed that the transformed expansion coefficients $\bar{y}_{n}$ should satisfy the condition:

$$
\frac{\mathcal{D} \bar{y}_{n}}{\mathcal{D} \bar{x}}=0
$$

(Remark: Note that Equation [13] in [1] contains a typing error: in the second term on the right-hand side, " $y_{n}$ " has to be replaced by " $\bar{y}_{n}$ "). However, a restriction such as Equation (63) would have severe consequences for the transformation rule of the variables $y(x) d x$ and $x$, and it is not advisable to enforce this unnecessary restriction; in particular, as this restriction is artificially generated in [1], namely by introducing a functional decomposition of $y(x) d x$ in Equation [10], which has, in the end, even no necessity in itself to successfully analyze the Hopf-Burgers Equation (3) for Lie-point symmetries (see e.g., our proposed approach in Section 2). To corroborate our claim, let us separately verify the relations of Equations [13] and [14] for the following transformation (up to first order), which was found as the symmetry solution $X_{4}^{\text {phys }}$ in [1] (p. 1561) for the unbounded domain $G=(-\infty,+\infty)$ :

$$
\left.\begin{array}{c}
\bar{t}=t+\varepsilon \cdot t^{2}, \quad \bar{x}=x+\varepsilon \cdot t x, \quad \overline{y(x) d x}=y(x) d x+\varepsilon \cdot t y(x) d x \\
\bar{\Phi}=\Phi+\varepsilon \cdot \frac{i}{2} \Phi \int x y(x) d x
\end{array}\right\}
$$

in order to see that this transformation is only compatible with Equation [13], but not to Equation [14]. The required transformation rule for the expansion coefficients $y_{n}$ is induced by Equation (64), which, for this particular configuration up to first order, all transform invariantly according to Equation (60) when using Equation (62):

$$
\bar{y}_{n}=y_{n}+\varepsilon \cdot t\left(\int_{G} h_{n}(x) y(x) d x-y_{n}\right)=y_{n}
$$

while the spatial 1D volume element transforms as:

$$
d \bar{x}=d x+\varepsilon \cdot t d x
$$

Now, since the left- and right-hand sides of Equation [13] evaluate to:

$$
\begin{aligned}
\operatorname{LHS}_{\text {Eq.[13] }}: & \frac{\overline{\mathcal{y}(x) d x}}{\mathcal{D} t}=\frac{\partial \overline{y(x) d x}}{\partial t}=\varepsilon \cdot y(x) d x+\mathcal{O}\left(\varepsilon^{2}\right) \\
\operatorname{RHS}_{\mathrm{Eq} \cdot[13]}: & \sum_{n=1}^{\infty} \frac{\mathcal{D} \bar{y}_{n}}{\mathcal{D} t} \bar{h}_{n}(\bar{x}) d \bar{x}+\sum_{n=1}^{\infty} \bar{y}_{n} \frac{\mathcal{D} \bar{h}_{n}(\bar{x}) d \bar{x}}{\mathcal{D} t}=\sum_{n=1}^{\infty} \bar{y}_{n} \frac{\partial \bar{h}_{n}(\bar{x}) d \bar{x}}{\partial t} \\
& =\sum_{n=1}^{\infty} \varepsilon \bar{y}_{n} h_{n}(x) d x+\mathcal{O}\left(\varepsilon^{2}\right)=\varepsilon \cdot y(x) d x+\mathcal{O}\left(\varepsilon^{2}\right)
\end{aligned}
$$


and the corresponding sides of Equation [14] to:

$$
\begin{aligned}
\operatorname{LHS}_{\mathrm{Eq} \cdot[14]}: & \sum_{n=1}^{\infty} \frac{\mathcal{D} \bar{y}_{n}}{\mathcal{D} t} \bar{h}_{n}(\bar{x}) d \bar{x}=0 \\
\mathrm{RHS}_{\mathrm{Eq} \cdot[14]}: & \frac{\mathcal{D} \overline{y(x) d x}}{\mathcal{D} t}-\frac{\mathcal{D} \overline{y(x) d x}}{\mathcal{D} \bar{x}} \frac{\mathcal{D} \bar{x}}{\mathcal{D} t}=\varepsilon \cdot y(x) d x-\varepsilon \cdot x \frac{\partial \overline{y(x) d x}}{\partial \bar{x}}+\mathcal{O}\left(\varepsilon^{2}\right) \\
& =\varepsilon \cdot\left(y(x)-x \partial_{x} y(x)\right) d x+\mathcal{O}\left(\varepsilon^{2}\right)
\end{aligned}
$$

we see that while Equation [13] is generally satisfied, Equation [14] is only satisfied for the special case when $y(x)=c \cdot x$, where $c$ is an arbitrary integration constant. But, since the considered transformation of Equation (64) is only defined in the unbounded domain $G=(-\infty,+\infty)$, the function $y(x)$ must be an asymptotically decaying function, $c f$. [13], which thus forces the integration constant to be $c=0$. That means we have to demand $y(x)=0$, but which, obviously, does not make any sense. In other words, the transformation of Equation (64) is not compatible to Equation [14], simply because it is not generally valid. (Remark: See Discussion E.1, in particular the transformation of Equation (59), for why Equation (68) is in fact the only correct formulation for the right-hand side of Equation [13] and not its formulation as given in [1]). To nonetheless allow for Equation [14], additional constraints for the infinitesimals $\xi_{y(x)} d x$ and $\xi_{x}$ must be placed. However, this has not been done in [1]; instead, Equation [14] is imposed as a generally valid relation without any restrictions, representing thus a technical error, which runs through the entire study. Additionally, to simply declare "that $t, x$ and the infinite set $\left\{y_{n}\right\}$ are the independent variables" [1] (p. 1545), such that $\mathcal{D} \bar{y}_{n} / \mathcal{D} \bar{x}=0$ of Equation (63) imposes no restrictions, is incorrect, because, when following their initial argument, the variables $t, x$ and $y(x) d x$ form the independent variables and not $t, x$ and $y_{n}$; see the above Discussion E.1, where we explicitly show that the expansion coefficients $y_{n}$ do not transform independently from the other variables. This confusion in the dependencies also brings us to the next issue.

E.3: The partial integration in Equation [15] to obtain Equation [16] is not justified. Since $\overline{y\left(x^{\prime}\right) d x^{\prime}}$ is identified or treated in [1] as an own independent variable next to $x^{\prime}$ (see the arguments, e.g., on p. 1545 and p. 1549), the relative variation of both variables in the untransformed, as well as in the transformed domain must be zero:

$$
\frac{\mathcal{D} y\left(x^{\prime}\right) d x^{\prime}}{\mathcal{D} x^{\prime}}=\frac{\mathcal{D} \overline{y\left(x^{\prime}\right) d x^{\prime}}}{\mathcal{D} \overline{x^{\prime}}}=0
$$

(Remark: Note that the total variation operator $\mathcal{D}$ only scans for explicit dependencies. Hence, for an overall consistent analysis, Equation (71) must already apply in Equation (70); but, even this consequence still has no effect on the incompatibility feature of Equation [14] in [1] with the transformation of Equation (64)).

Hence, when strictly following the arguments in [1], no partial integration in the last term of Equation [15] can be executed, which means that the appearance of the last term in Equation [16] is incorrect, in particular as it misleadingly suggests a non-zero contribution to this equation. The same problem one faces a page later when the expressions for the infinitesimals $\zeta_{; y(x)}$ and $\zeta_{; y(x) y(x)}$ get constructed, which all are incorrect, as they always involve too many terms. Note that the condition of Equation (71) is at the heart of every Lie-point symmetry analysis, in that if $\alpha$ and $\beta$ are independent variables, then their relative variation must be zero; analogously for the corresponding transformed variables $\bar{\alpha}$ and $\bar{\beta}$ :

$$
\frac{\mathcal{D} \alpha}{\mathcal{D} \beta}=\frac{\mathcal{D} \beta}{\mathcal{D} \alpha}=\frac{\mathcal{D} \bar{\alpha}}{\mathcal{D} \bar{\beta}}=\frac{\mathcal{D} \bar{\beta}}{\mathcal{D} \bar{\alpha}}=0
$$


E.4: The conclusion made in [1] (p. 1551), namely that if the multipoint velocity correlation functions vanish at infinity for an unbounded domain, then all functional derivatives of $\Phi$ vanish at infinity, too, is generally incorrect. In particular, their argument that if Equation [24] is imposed, i.e.,

$$
U^{t}(x= \pm \infty)=0
$$

that then the evaluation, e.g., of the first order functional derivative at infinity will give the zero result:

$$
\left.\Phi_{, y(x)}\right|_{x= \pm \infty}=\left.i\left\langle U^{t}(x) e^{i\left(U^{t}, y\right)}\right\rangle\right|_{x= \pm \infty}=0
$$

cannot be correct in the general sense, simply because the above coarse-graining operator \langle\rangle is not defined as a point-based ensemble operator, where the above reasoning would then be generally true, but as a function-based ensemble operator that is expressed as a path integral over all possible configuration states $U^{t}$ in the following way (see Equation [1] or Definition 2 in [1] (p. 1538)):

$$
\left\langle e^{i\left(U^{t}, y\right)}\right\rangle=\int e^{i(v(x), y)} f^{t}(v(x)) \mathfrak{D} v(x)
$$

where $f^{t}$ is the probability density functional and $\mathfrak{D} v(x)$ the functional volume element over an ensemble of all possible spatial velocity fields $v(x)$ (for more details, see, e.g., [13]). Hence, a point-wise evaluation as proposed in Equation (74) is ill-conditioned, i.e., to evaluate the following mathematical object:

$$
\left.\left\langle U^{t}(x) e^{i\left(U^{t}, y\right)}\right\rangle\right|_{x= \pm \infty}=\left[\int e^{i(v(x), y)} v(x) f^{t}(v(x)) \mathfrak{D} v(x)\right]_{x= \pm \infty}
$$

is in general not well defined, since a priori it is not clear how and in which way all functional elements within the path integral collectively behave when $x$ tends to infinity. To substantiate our argument, let us give a concrete example where the evaluation $\left.\Phi_{, y(x)}\right|_{x= \pm \infty}$ in Equation (74) is not zero. Consider, for example, the following well-defined specification of the functional $\Phi$ :

$$
\Phi\left(t, y\left(x^{\prime}\right)\right)=\exp \left(i f(t) \int d x^{\prime} y\left(x^{\prime}\right)\left(e^{-\lambda x^{\prime 2}}+\int d x^{\prime \prime} d x^{\prime \prime \prime} g\left(x^{\prime \prime}, x^{\prime \prime \prime}\right) y\left(x^{\prime \prime}\right) y\left(x^{\prime \prime \prime}\right)\right)\right)
$$

where $f$ is an arbitrary real function, $\lambda>0$ some positive real-valued constant and $g$ a two-dimensional real and symmetric scalar function decaying sufficiently fast at space infinity, i.e., $g(x):=g\left(x_{1}, x_{2}\right)=g\left(x_{2}, x_{1}\right) \rightarrow 0$ for $\|x\| \rightarrow \infty$. (Remark: Note that $y(x)$ is defined as a real function; see [13]). In the wording of [1], the specification of Equation (77) represents a physically-relevant functional, since it respects all the constraints of Definition 11 given in [1] (p. 1559):

$$
\Phi^{*}\left(t, y\left(x^{\prime}\right)\right)=\Phi\left(t,-y\left(x^{\prime}\right)\right), \quad \Phi(t, 0)=1, \quad\left|\Phi\left(t, y\left(x^{\prime}\right)\right)\right| \leq 1
$$

Now, by taking the first order functional derivative of Equation (77), we get:

$$
\Phi_{, y(x)}=\frac{\delta \Phi}{\delta y(x)}=i f(t) \cdot\left(e^{-\lambda x^{2}}+\int d x^{\prime} d x^{\prime \prime}\left(g\left(x^{\prime}, x^{\prime \prime}\right)+2 g\left(x, x^{\prime}\right)\right) y\left(x^{\prime}\right) y\left(x^{\prime \prime}\right)\right) \cdot \Phi
$$

When evaluating this result for $y(x)=0$, one yields the first order velocity correlation function (see Equation [5] in [1] (p. 1539))

$$
\left\langle U^{t}(x)\right\rangle=\left.\frac{1}{i} \Phi_{, y(x)}\right|_{y=0}=f(t) \cdot e^{-\lambda x^{2}}
$$


which then, when evaluated at the infinite far point:

$$
\left\langle U^{t}(x= \pm \infty)\right\rangle=\left[f(t) \cdot e^{-\lambda x^{2}}\right]_{x= \pm \infty}=0, \quad \lambda>0
$$

satisfies the correct imposed condition of Equation (73). Similarly, for all higher (multipoint) moments, we also get:

$$
\left.\left\langle U^{t}\left(x_{1}\right) \cdots U^{t}\left(x_{n}\right)\right\rangle\right|_{\|x\| \rightarrow \infty}=\left.\frac{1}{i^{n}} \Phi_{, y\left(x_{1}\right) \ldots y\left(x_{n}\right)}\right|_{y=0,\|x\| \rightarrow \infty}=0
$$

That means the specified functional of Equation (77) is compatible to the (instantaneous) velocity constraint of Equation (73) in that it induces the correctly-conditioned (statistical) multipoint moments. But, now, if we directly evaluate, e.g., the functional derivative of Equation (79) only at the infinite far point:

$$
\left.\Phi_{, y(x)}\right|_{x= \pm \infty}=\left[i f(t)\left(e^{-\lambda x^{2}}+\int d x^{\prime} d x^{\prime \prime}\left(g\left(x^{\prime}, x^{\prime \prime}\right)+2 g\left(x, x^{\prime}\right)\right) y\left(x^{\prime}\right) y\left(x^{\prime \prime}\right)\right) \Phi_{x= \pm \infty}\right.
$$

then it does not necessarily evaluate to zero if $y \neq 0$; in particular, it will evaluate to:

$$
\left.\Phi_{, y(x)}\right|_{x= \pm \infty}=i f(t) \Phi \int d x^{\prime} d x^{\prime \prime} g\left(x^{\prime}, x^{\prime \prime}\right) y\left(x^{\prime}\right) y\left(x^{\prime \prime}\right) \neq 0
$$

Hence, in contrast to the general claim of Equation (74) made in [1] (p. 1551), it is possible to construct a functional $\Phi$, e.g., Equation (77), which satisfies all physical constraints of Equation (78) and which induces the correct imposed condition of Equation (73), but which in general does not evaluate to zero when taking its functional derivative at infinity, as we can clearly see in Equation (84). This problem will then continue for all higher order functional derivatives. That means, the reasoning in [1] (p. 1551) "that all functional derivatives of $\Phi$ vanish for $x \rightarrow \pm \infty$ " if the condition of Equation [24], i.e., $U^{t}(x= \pm \infty)=0$ is imposed, is incorrect in the sense that this argument may not be used, e.g., to perform partial integrations, as it was generally done throughout [1].

Worthwhile to note here is that when looking at the structure of the Hopf-Burgers Equation (3) more closely, one can see that the functional derivatives do not even have to converge for $|x| \rightarrow \infty$. Since by definition, $y(x)$ must be an asymptotically-decaying function, $c f$. [13], the functional derivatives appearing in Equation (3) are thus allowed to diverge within a certain prescribed order. For example, if $y(x)$ decays as $1 / x^{n}$ for $|x| \rightarrow \infty$, then the (viscous) first-order functional derivative may diverge, but not faster than $x^{n}$ and the (inertial) second-order one not faster than $x^{n-1}$. This can be easily verified by power counting as within these assumptions, the integral operator over $x$ in Equation (3) is well-conditioned.

Author Contributions: Michael Frewer wrote the comment. George Khujadze carefully re-calculated and cross-validated all results and arguments appearing in this comment.

Conflicts of Interest: The authors declare no conflict of interest. 


\section{Appendix A. Proof that $X_{4}^{\text {phys }}-X_{6}^{\text {phys }}$ in [1] Are Not Admitted as Symmetry Transformations}

This part of the comment will prove that the following three global transformations associated with the generators $X_{4}^{\text {phys }}, X_{5}^{\text {phys }}$ and $X_{6}^{\text {phys }}$ as listed in Table 2 [1] (p. 1562):

$$
\begin{aligned}
& X_{4}^{\text {phys }} \rightarrow \mathrm{T}_{4}: \quad \bar{t}=\frac{t}{1-2 t \varepsilon}, \quad \bar{x}=\frac{x}{1-2 t \varepsilon}, \quad \bar{y}(\bar{x})=y(x), \quad \bar{\Phi}=\Phi \exp \left(i \frac{\varepsilon}{1-2 t \varepsilon} \int x^{\prime} y\left(x^{\prime}\right) d x^{\prime}\right) \\
& X_{5}^{\text {phys }} \rightarrow \mathrm{T}_{5}: \quad \bar{t}=t, \quad \bar{x}=x+2 t \varepsilon, \quad \bar{y}(\bar{x})=y(x), \quad \bar{\Phi}=\Phi \exp \left(i \varepsilon \int y\left(x^{\prime}\right) d x^{\prime}\right) \\
& X_{6}^{\text {phys }} \rightarrow \mathrm{T}_{6}: \quad \bar{t}=t, \quad \bar{x}=x, \quad \bar{y}(\bar{x})=y(x)+\varepsilon, \quad \bar{\Phi}=\Phi
\end{aligned}
$$

are not admitted as symmetry transformations by the considered functional Hopf-Burgers Equation (3), as it is incorrectly claimed in [1]; later on, the correct intermediate symmetry transformations will be given instead. By "intermediate", we refer to the fact that the symmetry analysis can be split into two subsequent steps: First we will perform the symmetry analysis in a more general setting, as it was also done in [1], namely by first excluding the internal Hopf-consistency condition $\partial_{t} y(x)=0$ from the analysis, which then, only in the second step, will then be included to eventually guarantee an overall consistent invariance analysis. This splitting has the advantage of following and understanding in each step the different symmetry-breaking mechanisms, which the Hopf-Burgers Equation (3) inherently owns. (Remark: Note that in $\mathrm{T}_{4}$ of Equation (A1) the (independent) variable $y$ transforms invariantly. The combined product $y \cdot d x$, where $d x$ is the 1D volume element (Jacobian), will then of course transform as given in Table 2 [1] (p. 1562), i.e., as $\overline{y \cdot d x}=\bar{y} \cdot d \bar{x}=\frac{y \cdot d x}{1-2 t \varepsilon}$ ).

In order to perform this first general step, it is necessary to know the inverse transformations of Equations (A1)-(A3). This is easily established, because, since they form additive Lie-groups, their inverse transformations can be readily read off as:

$$
\begin{array}{ll}
\mathrm{T}_{4}^{-1}: & t=\frac{\bar{t}}{1+2 \bar{t} \varepsilon}, \quad x=\frac{\bar{x}}{1+2 \bar{\epsilon} \varepsilon}, \quad y(x)=\bar{y}(\bar{x}), \quad \Phi=\bar{\Phi} \exp \left(-i \frac{\varepsilon}{1+2 \bar{\epsilon} \varepsilon} \int \bar{x}^{\prime} \bar{y}\left(\bar{x}^{\prime}\right) d \bar{x}^{\prime}\right) \\
\mathrm{T}_{5}^{-1}: & t=\bar{t}, \quad x=\bar{x}-2 \bar{t} \varepsilon, \quad y(x)=\bar{y}(\bar{x}), \quad \Phi=\bar{\Phi} \exp \left(-i \varepsilon \int \bar{y}\left(\bar{x}^{\prime}\right) d \bar{x}^{\prime}\right) \\
\mathrm{T}_{6}^{-1}: & t=\bar{t}, \quad x=\bar{x}, \quad y(x)=\bar{y}(\bar{x})-\varepsilon, \quad \Phi=\bar{\Phi}
\end{array}
$$

Important to note is that when we transform Equation (3), we are dealing with three independent variables $t, x$ and $y$ and one dependent variable $\Phi$, which does not explicitly depend on the spatial coordinate $x$, i.e., in both the "un-bared" and "bared" domain, we have to respect the conditions $\partial_{x} \Phi=0$ and $\partial_{\bar{x}} \bar{\Phi}=0$, respectively, where in each case, the partial derivative is a context-related derivative, which only scans for explicit spatial dependencies (see also Discussion (b) in Section 1).

In order to transform the Hopf-Burgers Equation (3), we first have to determine the transformation rules for the following partial and functional derivative operators relative to the independent variables $t, x$ and $y$, which we can achieve by applying the chain rule:

$$
\begin{aligned}
\frac{\partial}{\partial \bar{t}} & =\frac{\partial t}{\partial \bar{t}} \frac{\partial}{\partial t}+\frac{\partial x}{\partial \bar{t}} \frac{\partial}{\partial x}+\int d x \frac{\partial y(x)}{\partial \bar{t}} \frac{\delta}{\delta y(x)} \\
\frac{\partial}{\partial \bar{x}} & =\frac{\partial t}{\partial \bar{x}} \frac{\partial}{\partial t}+\frac{\partial x}{\partial \bar{x}} \frac{\partial}{\partial x}+\int d x \frac{\partial y(x)}{\partial \bar{x}} \frac{\delta}{\delta y(x)} \\
\frac{\delta}{\delta \bar{y}(\bar{x})} & =\frac{\delta t}{\delta \bar{y}(\bar{x})} \frac{\partial}{\partial t}+\frac{\delta x}{\delta \bar{y}(\bar{x})} \frac{\partial}{\partial x}+\int d x^{\prime \prime} \frac{\delta y\left(x^{\prime \prime}\right)}{\delta \bar{y}(\bar{x})} \frac{\delta}{\delta y\left(x^{\prime \prime}\right)}
\end{aligned}
$$

(Remark: The chain rule as given by Equations (A7)-(A9) is complete, since the transformations of Equations (A1)-(A3) for the independent variables $t \mapsto \bar{t}, x \mapsto \bar{x}$ and $y \mapsto \bar{y}$ do not depend on 
the variable $\Phi)$. The transformation rules for all higher order differential operators will then follow accordingly. The crucial point here is to recognize that the partial derivatives in Equations (A7)-(A9) are context-related in the sense that they only scan for explicit dependencies. Hence, when inserting the specific (inverse) transformations of Equations (A4)-(A6) into the above expressions, they will reduce to:

$$
\begin{aligned}
\frac{\partial}{\partial \bar{t}} & =\frac{\partial t}{\partial \bar{t}} \frac{\partial}{\partial t}+\frac{\partial x}{\partial \bar{t}} \frac{\partial}{\partial x} \\
\frac{\partial}{\partial \bar{x}} & =\frac{\partial t}{\partial \bar{x}} \frac{\partial}{\partial t}+\frac{\partial x}{\partial \bar{x}} \frac{\partial}{\partial x} \\
\frac{\delta}{\delta \bar{y}(\bar{x})} & =\int d x^{\prime \prime} \frac{\delta y\left(x^{\prime \prime}\right)}{\delta \bar{y}(\bar{x})} \frac{\delta}{\delta y\left(x^{\prime \prime}\right)}
\end{aligned}
$$

since for all three transformations $\mathrm{T}_{4}^{-1}, \mathrm{~T}_{5}^{-1}$ and $\mathrm{T}_{6}^{-1}$ given by Equations (A4)-(A6), the functional transformation $y \mapsto \bar{y}$ does not explicitly depend on $\bar{t}$ and $\bar{x}$, and, the coordinate transformations $t \mapsto \bar{t}$ and $x \mapsto \bar{x}$ not on $\bar{y}$.

\section{A.1. Transformation $\mathrm{T}_{4}$}

In the specific case of $T_{4}$ and its inverse $T_{4}^{-1}$ in Equation (A4), the transformation rules for all relevant partial and functional derivatives appearing in the Hopf-Burgers Equation (3) then take, according to Equations (A10)-(A12), the specific form:

$$
\begin{aligned}
\frac{\partial}{\partial \bar{t}} & =(1-2 t \varepsilon)^{2} \frac{\partial}{\partial t}-2 x \varepsilon(1-2 t \varepsilon) \frac{\partial}{\partial x} \\
\frac{\partial}{\partial \bar{x}} & =(1-2 t \varepsilon) \frac{\partial}{\partial x} \\
\frac{\delta}{\delta \bar{y}(\bar{x})} & =\int d x^{\prime \prime} \frac{\delta y\left(x^{\prime \prime}\right)}{\delta \bar{y}(\bar{x})} \frac{\delta}{\delta y\left(x^{\prime \prime}\right)} \\
& =\int \frac{d \bar{x}^{\prime \prime}}{1+2 \bar{t} \varepsilon} \frac{\delta \bar{y}\left(\bar{x}^{\prime \prime}\right)}{\delta \bar{y}(\bar{x})} \frac{\delta}{\delta y\left(\frac{\bar{x}^{\prime \prime}}{1+2 \overline{t \varepsilon}}\right)}=(1-2 t \varepsilon) \int d \bar{x}^{\prime \prime} \delta\left(\bar{x}^{\prime \prime}-\bar{x}\right) \frac{\delta}{\delta y\left(\frac{\bar{x}^{\prime \prime}}{1+2 \bar{t} \varepsilon}\right)} \\
& =(1-2 t \varepsilon) \frac{\delta}{\delta y\left(\frac{\bar{x}}{1+2 \bar{t}_{\varepsilon}}\right)}=(1-2 t \varepsilon) \frac{\delta}{\delta y(x)}
\end{aligned}
$$

and for the relevant second order differential operators, the form:

$$
\begin{gathered}
\frac{\partial^{2}}{\partial \bar{x}^{2}}=(1-2 t \varepsilon)^{2} \frac{\partial^{2}}{\partial x^{2}} \\
\frac{\delta^{2}}{\delta \bar{y}(\bar{x}) \delta \bar{y}(\bar{x})}=(1-2 t \varepsilon)^{2} \frac{\delta^{2}}{\delta y(x) \delta y(x)}
\end{gathered}
$$


Please note that instead of transforming the volume element $d x^{\prime \prime}$, one can in this particular case also derive the transformation rule in Equation (A15) by making use of the scaling property of the Dirac delta function:

$$
\begin{aligned}
\frac{\delta}{\delta \bar{y}(\bar{x})} & =\int d x^{\prime \prime} \frac{\delta y\left(x^{\prime \prime}\right)}{\delta \bar{y}(\bar{x})} \frac{\delta}{\delta y\left(x^{\prime \prime}\right)} \\
& =\int d x^{\prime \prime} \frac{\delta \bar{y}\left(\bar{x}^{\prime \prime}\right)}{\delta \bar{y}(\bar{x})} \frac{\delta}{\delta y\left(x^{\prime \prime}\right)}=\int d x^{\prime \prime} \delta\left(\bar{x}^{\prime \prime}-\bar{x}\right) \frac{\delta}{\delta y\left(x^{\prime \prime}\right)}=\int d x^{\prime \prime} \delta\left(\frac{1}{1-2 t \varepsilon} \cdot\left(x^{\prime \prime}-x\right)\right) \frac{\delta}{\delta y\left(x^{\prime \prime}\right)} \\
& =(1-2 t \varepsilon) \int d x^{\prime \prime} \delta\left(x^{\prime \prime}-x\right) \frac{\delta}{\delta y\left(x^{\prime \prime}\right)}=(1-2 t \varepsilon) \frac{\delta}{\delta y(x)}
\end{aligned}
$$

By applying Equation (A13) onto $\bar{\Phi}$ of Equation (A1) and by introducing the functional:

$$
\gamma=\gamma\left(y\left(x^{\prime}\right), t\right)=\exp \left(i \frac{\varepsilon}{1-2 t \varepsilon} \int x^{\prime} y\left(x^{\prime}\right) d x^{\prime}\right)
$$

we yield, by knowing that $\partial_{x} \Phi=0$, the following transformation for the temporal part on the left-hand side of the Hopf-Burgers Equation (3) as:

$$
\begin{aligned}
\frac{\partial \bar{\Phi}}{\partial \bar{t}} & =(1-2 t \varepsilon)^{2} \cdot\left(\gamma \cdot \frac{\partial \Phi}{\partial t}+\Phi \cdot \frac{\partial \gamma}{\partial t}\right)-2 x \varepsilon(1-2 t \varepsilon) \cdot \gamma \cdot \frac{\partial \Phi}{\partial x} \\
& =(1-2 t \varepsilon)^{2} \cdot \gamma \cdot\left(\frac{\partial \Phi}{\partial t}+i \frac{2 \varepsilon^{2}}{(1-2 t \varepsilon)^{2}} \cdot \Phi \cdot \int x^{\prime} y\left(x^{\prime}\right) d x^{\prime}\right) \\
& =(1-2 t \varepsilon)^{2} \cdot \gamma \cdot \frac{\partial \Phi}{\partial t}+2 i \varepsilon^{2} \cdot \gamma \cdot \Phi \cdot \int x^{\prime} y\left(x^{\prime}\right) d x^{\prime}
\end{aligned}
$$

Note that the above transformation of Equation (A20) can also be derived by the following, alternative argument, namely by only looking iteratively at the dependencies of the given transformation $\bar{\Phi}$ in $\mathrm{T}_{4}$ of Equation (A1):

$$
\begin{aligned}
\bar{\Phi} & =\bar{\Phi}(\Phi, y, t) \\
& =\bar{\Phi}(\Phi(y, t), y, t) \\
& =\bar{\Phi}(\Phi(y(\bar{y}), t(\bar{t})), y(\bar{y}), t(\bar{t}))
\end{aligned}
$$

where the first line shows that the transformation $\bar{\Phi}$ depends on $\Phi, y$ and $t$, the second line that $\Phi$ is only a function of $y$ and $t$ and the last line where the transformations for $y$ and $t$ are finally inserted, which, in this particular case $T_{4}$ of Equation (A1), only depend on $\bar{y}$ and $\bar{t}$, respectively. Taking then the time derivative of Equation (A21) and respecting the condition $\partial_{\bar{t}} \bar{y}=0$ in knowing that $y$ transforms invariantly, i.e., $\bar{y}=y$, we obtain the identical result to Equation (A20):

$$
\begin{aligned}
\frac{\partial \bar{\Phi}}{\partial \bar{t}} & =\frac{\partial \Phi}{\partial \bar{t}} \frac{\partial \bar{\Phi}}{\partial \Phi}+\int d x^{\prime} \frac{\partial y\left(x^{\prime}\right)}{\partial \bar{t}} \frac{\delta \bar{\Phi}}{\delta y\left(x^{\prime}\right)}+\frac{\partial t}{\partial \bar{t}} \frac{\partial \bar{\Phi}}{\partial t} \\
& =\frac{\partial \bar{\Phi}}{\partial \Phi}\left(\frac{\partial t}{\partial \bar{t}} \frac{\partial \Phi}{\partial t}+\int d x^{\prime} \frac{\partial y\left(x^{\prime}\right)}{\partial \bar{t}} \frac{\delta \Phi}{\delta y\left(x^{\prime}\right)}\right)+\int d x^{\prime} \frac{\partial y\left(x^{\prime}\right)}{\partial \bar{t}} \frac{\delta \bar{\Phi}}{\delta y\left(x^{\prime}\right)}+\frac{\partial t}{\partial \bar{t}} \frac{\partial \bar{\Phi}}{\partial t} \\
& =\frac{\partial \bar{\Phi}}{\partial \Phi} \frac{\partial t}{\partial \bar{t}} \frac{\partial \Phi}{\partial t}+\frac{\partial t}{\partial \bar{t}} \frac{\partial \bar{\Phi}}{\partial t}
\end{aligned}
$$


The last step in this process is to determine the transformation of the inertial and viscous part on the right-hand side of the Hopf-Burgers Equation (3), which according to Equations (A14)-(A17) and Equation (A1) transforms as:

$$
\begin{aligned}
\int \bar{y}(\bar{x}) & \left(i \frac{\partial}{\partial \bar{x}} \frac{\delta^{2} \bar{\Phi}}{\delta \bar{y}(\bar{x}) \delta \bar{y}(\bar{x})}+v \frac{\partial^{2}}{\partial \bar{x}^{2}} \frac{\delta \bar{\Phi}}{\delta \bar{y}(\bar{x})}\right) d \bar{x} \\
= & \int y(x)\left(i(1-2 t \varepsilon)^{3} \frac{\partial}{\partial x} \frac{\delta^{2} \bar{\Phi}}{\delta y(x) \delta y(x)}+v(1-2 t \varepsilon)^{3} \frac{\partial^{2}}{\partial x^{2}} \frac{\delta \bar{\Phi}}{\delta y(x)}\right) \frac{d x}{1-2 t \varepsilon} \\
= & (1-2 t \varepsilon)^{2} \int y(x)\left(i \frac{\partial}{\partial x}\left[\frac{\delta^{2} \gamma}{\delta y(x) \delta y(x)} \Phi+2 \frac{\delta \gamma}{\delta y(x)} \frac{\delta \Phi}{\delta y(x)}+\gamma \frac{\delta^{2} \Phi}{\delta y(x) \delta y(x)}\right]\right. \\
& \left.+v \frac{\partial^{2}}{\partial x^{2}}\left[\frac{\delta \gamma}{\delta y(x)} \Phi+\gamma \frac{\delta \Phi}{\delta y(x)}\right]\right) d x \\
= & (1-2 t \varepsilon)^{2} \cdot \gamma \cdot \int y(x)\left(i \frac{\partial}{\partial x}\left[\frac{(i \varepsilon x)^{2}}{(1-2 t \varepsilon)^{2}} \Phi+\frac{2 i \varepsilon x}{1-2 t \varepsilon} \frac{\delta \Phi}{\delta y(x)}+\frac{\delta^{2} \Phi}{\delta y(x) \delta y(x)}\right]\right. \\
= & (1-2 t \varepsilon)^{2} \cdot \gamma \cdot \int y(x)\left(i \frac{\partial}{\partial x} \frac{\delta^{2} \Phi}{\delta y(x) \delta y(x)}+v \frac{\delta^{2}}{\partial x^{2}} \frac{\delta \Phi}{\delta y(x)}\right) d x \\
& \quad 2 i \varepsilon^{2} \cdot \gamma \cdot \Phi \cdot \int x y(x) d x-2 \varepsilon(1-2 t \varepsilon) \cdot \gamma \cdot \int y(x)\left(\frac{\delta \Phi}{\delta y(x)}+x \frac{\partial}{\partial x} \frac{\delta \Phi}{\delta y(x)}\right) d x
\end{aligned}
$$

where in the first term of the last line, the functional $\Phi$ could be pulled out of the integral for $x$, since the dependence of $\Phi$ in $\mathrm{T}_{4}$ of Equation (A1) was initially set up as $\Phi=\Phi\left(y\left(x^{\prime}\right), t\right)$ with an implicit dependence on $x^{\prime}$ (and not on $x$ ), in order to perform all functional derivatives consistently.

Now, when comparing the transformed left-hand side of Equation (A20) with the transformed right-hand side of Equation (A23) for the Hopf-Burgers Equation (3), we see that the transformation $\mathrm{T}_{4}$ of Equation (A1) is not admitted as a symmetry, since the considered equation does not transform invariantly, i.e.,

$$
\begin{aligned}
0=\frac{\partial \bar{\Phi}}{\partial \bar{t}}-\int \bar{y}(\bar{x})\left(i \frac{\partial}{\partial \bar{x}} \frac{\delta^{2} \bar{\Phi}}{\delta \bar{y}(\bar{x}) \delta \bar{y}(\bar{x})}+v \frac{\partial^{2}}{\partial \bar{x}^{2}} \frac{\delta \bar{\Phi}}{\delta \bar{y}(\bar{x})}\right) d \bar{x} & \\
& \neq \frac{\partial \Phi}{\partial t}-\int y(x)\left(i \frac{\partial}{\partial x} \frac{\delta^{2} \Phi}{\delta y(x) \delta y(x)}+v \frac{\partial^{2}}{\partial x^{2}} \frac{\delta \Phi}{\delta y(x)}\right) d x
\end{aligned}
$$

Instead, the correct intermediate statistical Hopf-symmetry corresponding to the projective symmetry:

$$
\mathrm{S}_{1}: \quad \bar{t}=\frac{t}{1-\varepsilon t^{\prime}}, \quad \bar{x}=\frac{x}{1-\varepsilon t^{\prime}}, \quad \overline{U^{t}}=(1-\varepsilon t) \cdot U^{t}+\varepsilon x
$$

of the deterministic 1D viscous Burgers equation (in non-conservative form; see [1] (p. 1540)):

$$
\frac{\partial U^{t}}{\partial t}+U^{t} \frac{\partial U^{t}}{\partial x}=v \frac{\partial^{2} U^{t}}{\partial x^{2}}
$$

is given by:

$$
\left\langle\mathrm{S}_{1}\right\rangle: \quad \bar{t}=\frac{t}{1-\varepsilon t^{\prime}}, \quad \bar{x}=\frac{x}{1-\varepsilon t}, \quad \bar{y}(\bar{x})=y(x), \quad \bar{\Phi}=\Phi
$$


which, as can be straightforwardly checked with the transformations rules given above, is admitted as a symmetry transformation by the Hopf-Burgers equation in the form:

$$
\frac{\partial \Phi}{\partial t}=\int y(x)\left(i \lim _{x^{\prime} \rightarrow x} \frac{\partial}{\partial x} \frac{\delta^{2} \Phi}{\delta y(x) \delta y\left(x^{\prime}\right)}+v \frac{\partial^{2}}{\partial x^{2}} \frac{\delta \Phi}{\delta y(x)}\right) d x
$$

as well as in the form:

$$
\frac{\partial \Phi}{\partial t}=\int y(x)\left(i \frac{\partial}{\partial x} \frac{\delta^{2} \Phi}{\delta y(x) \delta y(x)}+v \frac{\partial^{2}}{\partial x^{2}} \frac{\delta \Phi}{\delta y(x)}\right) d x
$$

To intuitively understand why in particular transformation $\left\langle\mathrm{S}_{1}\right\rangle$ of Equation (A27) is admitted as a symmetry and not $T_{4}$ of Equation (A1), one has to go back to Hopf's seminal study [13] and notice that the derivation of Equation (A29) was done under the premiss that the deterministic velocity field $U^{t}$ has to be zero at the boundary of the domain. Since $T_{4}$ of Equation (A1) was derived for the unbounded and thus infinite domain (see [1] (p. 1551)), it therefore is only compatible with Equation (A29) if the boundary values $U^{t}( \pm \infty)=0$ are mapped again to zero values at the infinite far boundary point $\overline{U^{t}}( \pm \infty)=0$. However, this is not given for the transformation of Equation (A25), since the transformed deterministic field and all of its multipoint correlations diverge at the infinite far boundary point, due to the existence of the non-decaying term $\varepsilon x$ in the transformation. That means, the transformed velocity field $\overline{U^{t}}$ in Equation (A25) is not an integrable function and, thus, not an element of the $L^{2}$-space anymore, as it was initially assumed in [1] (p. 1540). Hence, the transformation for $\overline{U^{t}}$ in Equation (A25) may not be used to transform, for example, the defining relation of Equation [1] in [1] (p. 1538) (see also Equation (75) for our redefined notation):

$$
\Phi(y(x), t)=\int e^{i \int y(x) U(x) d x} f(U(x), t) \mathfrak{D} U(x)
$$

upon which Equation (A29) is based. Because, if we would force the transformation of Equation (A25) onto Equation (A30) and assume that $y$ transforms invariantly and the probability density functional as $\bar{f}=f /(1-\varepsilon t)$, then we would obtain the result:

$$
\begin{aligned}
\bar{\Phi}(\bar{y}, \bar{t}) & =\int e^{i \int \bar{y} \bar{U} d \bar{x}} \bar{f}(\bar{U}, \bar{t}) \mathfrak{D} \bar{U}=\int e^{i \int y[(1-\varepsilon t) U+\varepsilon x] \frac{d x}{1-\varepsilon t} \bar{f}}(\bar{U}, \bar{t})(1-\varepsilon t) \mathfrak{D U} \\
& =e^{i \frac{\varepsilon}{1-\varepsilon \varepsilon} \int x y(x) d x} \int e^{i \int y U d x} f(U, t) \mathfrak{D U}=e^{i \frac{\varepsilon}{1-t \varepsilon} \int x y(x) d x} \cdot \Phi(y, t)
\end{aligned}
$$

which, up to a scaling factor of two in $t$, coincides with the transformation for $\bar{\Phi}$ from $T_{4}$ of Equation (A1). To note is that the difference in scaling by a factor two has its origin in the fact that the authors in [1] (p. 1540) considered, instead of Equation (A26), the temporally-rescaled conservative deterministic Burgers equation in Equation [6]:

$$
\frac{\partial U^{t}}{\partial t^{*}}+\frac{\partial\left(U^{t}\right)^{2}}{\partial x}=v^{*} \frac{\partial^{2} U^{t}}{\partial x^{2}}
$$

which admits the rescaled $t \mapsto t^{*}=t / 2$ projective symmetry of Equation (A25):

$$
\mathrm{S}_{1}^{*}: \quad \bar{t}^{*}=\frac{t^{*}}{1-2 \varepsilon t^{*}}, \quad \bar{x}=\frac{x}{1-2 \varepsilon t^{*}}, \quad \overline{U^{t}}=\left(1-2 \varepsilon t^{*}\right) \cdot U^{t}+\varepsilon x
$$

But, as we have proven above, the transformation, where $\Phi$ transforms as in Equation (A31), is not admitted as a symmetry by the Hopf-Burgers Equation (A29). The reason is simply that the transformation of Equation (A31) is not compatible with the internal boundary condition of Equation (A29), where all fields, also the transformed ones, have to be integrable functions decaying 
sufficiently fast at infinity. Instead, the transformed characteristic functional $\bar{\Phi}$ of Equation (A31) induces multipoint velocity correlations (Equation [5] in [1] (p. 1539)):

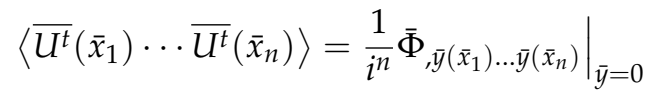

which diverge at the infinite far boundary point. Hence, in order to satisfy its internal boundary condition, the statistical Hopf-Burgers equation breaks the deterministic symmetry $\mathrm{S}_{1}$ of Equation (A25) in its underlying 1D Burgers Equation (A26) down to the statistical symmetry $\left\langle\mathrm{S}_{1}\right\rangle$ of Equation (A27), where then, $\Phi$ transforms invariantly. The same problem we also face with the deterministic Galilei invariance, but only in physical space, not in wavenumber space, as we will discuss in the next section.

Up to now, we only performed the symmetry analysis without including the consistency condition $\partial_{t} y(x)=0$. However, this condition (see Discussion (c) in Section 1) is absolutely necessary to really warrant an overall consistent symmetry analysis for the Hopf-Burgers Equation (A29). Hence, any symmetry admitted by Equation (A29) must be compatible with this condition that the independent functional variable $y$ only depends on $x$ and not on $t$. However, the symmetry $\left\langle\mathrm{S}_{1}\right\rangle$ of Equation (A27) and, thus, also the transformation $\mathrm{T}_{4}$ of Equation (A1) are not compatible with this condition, which, after all, can be straightforwardly seen, since:

$$
0=\partial_{t} y(x)=\partial_{t} \bar{y}(\bar{x})=\partial_{t} \bar{y}\left(\frac{x}{1-2 t \varepsilon}\right) \neq 0, \quad \text { if } \bar{y} \neq 0
$$

In other words, the condition $\partial_{t} y(x)=0$ finally breaks the intermediate symmetry $\left\langle\mathrm{S}_{1}\right\rangle$ of Equation (A27) down to a featureless identity transformation. Consequently, the statistical Hopf-Burgers Equation (A29) ultimately does not allow for a corresponding projective symmetry as in its underlying deterministic Equation (A26).

\section{A.2. Transformation $T_{5}$}

The proof that the transformation $T_{5}$ of Equation (A2) is not admitted as a symmetry by the Hopf-Burgers Equation (A29) runs completely analogous to the proof done in the previous section for $\mathrm{T}_{4}$ of Equation (A1). According to Equations (A10)-(A12), the transformation rules for all relevant partial and functional derivations now have the form:

$$
\left.\begin{array}{c}
\frac{\partial}{\partial \bar{t}}=\frac{\partial}{\partial t}-2 \varepsilon \frac{\partial}{\partial x}, \quad \frac{\partial}{\partial \bar{x}}=\frac{\partial}{\partial x}, \quad \frac{\partial^{2}}{\partial \bar{x}^{2}}=\frac{\partial^{2}}{\partial x^{2}} \\
\frac{\delta}{\delta \bar{y}(\bar{x})}=\frac{\delta}{\delta y(x)}, \quad \frac{\delta^{2}}{\delta \bar{y}(\bar{x}) \delta \bar{y}(\bar{x})}=\frac{\delta^{2}}{\delta y(x) \delta y(x)}
\end{array}\right\}
$$

which, when introducing the functional:

$$
\rho=\rho\left(y\left(x^{\prime}\right)\right)=\exp \left(i \varepsilon \int y\left(x^{\prime}\right) d x^{\prime}\right)
$$

will transform the temporal part on the left-hand side of the considered functional Hopf-Burgers Equation (A29) as:

$$
\frac{\partial \bar{\Phi}}{\partial \bar{t}}=\rho \frac{\partial \Phi}{\partial t}-2 \rho \varepsilon \frac{\partial \Phi}{\partial x}=\rho \frac{\partial \Phi}{\partial t}
$$


and the inertial and viscous part on its right-side as:

$$
\begin{aligned}
\int \bar{y}(\bar{x})( & \left.i \frac{\partial}{\partial \bar{x}} \frac{\delta^{2} \bar{\Phi}}{\delta \bar{y}(\bar{x}) \delta \bar{y}(\bar{x})}+v \frac{\partial^{2}}{\partial \bar{x}^{2}} \frac{\delta \bar{\Phi}}{\delta \bar{y}(\bar{x})}\right) d \bar{x} \\
= & \int y(x)\left(i \frac{\partial}{\partial x} \frac{\delta^{2} \bar{\Phi}}{\delta y(x) \delta y(x)}+v \frac{\partial^{2}}{\partial x^{2}} \frac{\delta \bar{\Phi}}{\delta y(x)}\right) d x \\
= & \int y(x)\left(i \frac{\partial}{\partial x}\left[\frac{\delta^{2} \rho}{\delta y(x) \delta y(x)} \Phi+2 \frac{\delta \rho}{\delta y(x)} \frac{\delta \Phi}{\delta y(x)}+\rho \frac{\delta^{2} \Phi}{\delta y(x) \delta y(x)}\right]\right. \\
& \left.\quad+v \frac{\partial^{2}}{\partial x^{2}}\left[\frac{\delta \rho}{\delta y(x)} \Phi+\rho \frac{\delta \Phi}{\delta y(x)}\right]\right) d x \\
= & \rho \int y(x)\left(i \frac{\partial}{\partial x}\left[(i \varepsilon)^{2} \Phi+2 i \varepsilon \frac{\delta \Phi}{\delta y(x)}+\frac{\delta^{2} \Phi}{\delta y(x) \delta y(x)}\right]+v \frac{\partial^{2}}{\partial x^{2}}\left[i \varepsilon \Phi+\frac{\delta \Phi}{\delta y(x)}\right]\right) d x \\
= & \rho \int y(x)\left(i \frac{\partial}{\partial x} \frac{\delta^{2} \Phi}{\delta y(x) \delta y(x)}+v \frac{\partial^{2}}{\partial x^{2}} \frac{\delta \Phi}{\delta y(x)}\right) d x-2 \rho \varepsilon \int y(x) \frac{\partial}{\partial x} \frac{\delta \Phi}{\delta y(x)} d x
\end{aligned}
$$

Equating now the transformed left-hand side of Equation (A38) with the transformed right-hand side of Equation (A39), we clearly observe that the Hopf-Burgers Equation (A29) does not stay invariant under the transformation $T_{5}$ of Equation (A2), due to the appearance of the last term in Equation (A39). Hence, the transformation $T_{5}$ of Equation (A2) is not admitted as a symmetry. Instead, the correct intermediate statistical Hopf-symmetry corresponding to the Galilei symmetry of the deterministic Burgers Equation (A26) in physical space :

$$
\mathrm{S}_{2}: \quad \bar{t}=t, \quad \bar{x}=x+t \varepsilon, \quad \overline{U^{t}}=U^{t}+\varepsilon
$$

is given by:

$$
\left\langle\mathrm{S}_{2}\right\rangle: \quad \bar{t}=t, \quad \bar{x}=x+t \varepsilon, \quad \bar{y}(\bar{x})=y(x), \quad \bar{\Phi}=\Phi
$$

As was observed and discussed in detail at the end of the previous Section A.1, we see again the effect in how the statistical Hopf-Burgers equation is also breaking the deterministic Galilei symmetry $\mathrm{S}_{2}$ of Equation (A40) in its underlying Burgers Equation (A26) down to the statistical symmetry $\left\langle\mathrm{S}_{2}\right\rangle$ of Equation (A41), where $\Phi$ then only transforms invariantly. The reason is again that the transformed defining relation of Equation (A30):

$$
\begin{aligned}
\bar{\Phi}(\bar{y}, \bar{t}) & =\int e^{i \int \bar{y} \bar{U} d \bar{x}} \bar{f}(\bar{U}, \bar{t}) \mathfrak{D} \bar{U}=\int e^{i \int y(U+\varepsilon) d x} f(U, t) \mathfrak{D} U \\
& =e^{i \varepsilon \int y(x) d x} \cdot \Phi(y, t)
\end{aligned}
$$

is generating multipoint velocity correlation functions, which are non-zero at the infinite far boundary point, if $\varepsilon \neq 0$, and, thus, violating again the internal boundary condition of the Hopf-Burgers Equation (A29). (Remark: In Equation (A42) we assumed that the fields $y$ and $f$ transform invariantly, which then coincides with the transformation for $\bar{\Phi}$ in $T_{5}$ of Equation (A2)).

Interesting to note here is that this breaking only occurs in physical space and not in wavenumber space. The simple reason is that for the Fourier transform of the deterministic Galilei symmetry of Equation (A40):

$$
\widehat{\mathrm{S}}_{2}: \quad \bar{t}=t, \quad \bar{k}=k, \quad \widehat{V}^{t}=e^{i k t \varepsilon} \widehat{V}^{t}+\varepsilon e^{i k t \varepsilon} \delta(k)
$$

where $k$ is the wavenumber variable and $\widehat{V}^{t}$ the Fourier transform of the velocity field $U^{t}$ :

$$
\widehat{V}^{t}=\int e^{i k x} U^{t} d x
$$


the transformed fields $\widehat{V}^{t}$ in an unbounded domain remain to be integrable functions if the corresponding untransformed fields $\widehat{V}^{t}$ are given as integrable functions in that domain. Hence, the transformed defining relation in unbounded wavenumber space (as defined in [13] (p. 105)):

$$
\overline{\widehat{\Phi}}(\bar{z}, \bar{t})=\int e^{i \int \bar{z} \bar{V}^{*} d \bar{k}} \bar{f}(\bar{V}, \bar{t}) \mathfrak{D} \bar{V}=e^{i \varepsilon z(0)} \cdot \widehat{\Phi}(z, t)
$$

where we assumed that the wavenumber field $z$ transforms as $\bar{z}=e^{i k t \varepsilon} z$ and the spectral probability density functional as $\overline{\hat{f}}=e^{-i k t \varepsilon} \widehat{f}$, generates integrable multipoint velocity correlation functions. In contrast to the unbounded physical domain, the transformation for $\bar{\Phi}$ in Equation (A45) is not broken in the corresponding statistical Hopf-symmetry to Equation (A43), which is:

$$
\left\langle\widehat{S}_{2}\right\rangle: \quad \bar{t}=t, \quad \bar{k}=k, \quad \bar{z}=e^{i k t \varepsilon} z, \quad \bar{\Phi}=e^{i \varepsilon z(0)} \widehat{\Phi}
$$

and which is admitted as an intermediate symmetry by the functional Hopf-Burgers equation in wavenumber space. Note therefore that it is due to all of these reasons that Hopf in his seminal study [13] preferred the spectral space over the physical space when exploring unbounded turbulent flows.

But, as soon as one includes the still remaining consistency conditions $\partial_{t} y(x)=0$ and $\partial_{t} z(k)=0$ into the above analysis in either space, the intermediate Galilei symmetry in physical space $\left\langle\mathrm{S}_{2}\right\rangle$ of Equation (A41), as well as in spectral space $\left\langle\widehat{S}_{2}\right\rangle$ of Equation (A46) will ultimately get broken. As in the previous Section A.1, the reason is again that both of these symmetries, Equations (A41) and (A46), are not compatible with the time-independence condition of $y(x)$ and $z(k)$, respectively, since obviously:

$$
\begin{aligned}
& 0=\partial_{t} y(x)=\partial_{t} \bar{y}(\bar{x})=\partial_{t} \bar{y}(x+t \varepsilon) \neq 0, \quad \text { if } \bar{y} \neq 0 \\
& 0=\partial_{t} z(k)=\partial_{t}\left(e^{-i k t \varepsilon} \bar{z}(\bar{k})\right)=-i k \varepsilon \cdot e^{-i k t \varepsilon} \bar{z}(k) \neq 0, \quad \text { if } \quad \bar{z} \neq 0
\end{aligned}
$$

\section{A.3. Transformation $\mathrm{T}_{6}$}

The argument that the transformation $T_{6}$ of Equation (A3) is not admitted as a symmetry by the Hopf-Burgers Equation (A29) is different from the ones shown in the previous sections for $T_{4}$ and $T_{5}$. Since all relevant partial and functional derivatives transform invariantly under $T_{6}$ of Equation (A3), which only marks a constant translation in $y$, the left-hand side of Equation (A29) thus transforms invariantly, as well:

$$
\frac{\partial \bar{\Phi}}{\partial \bar{t}}=\frac{\partial \Phi}{\partial t}
$$

while the right-hand side transforms as:

$$
\begin{aligned}
\int \bar{y}(\bar{x})\left(i \frac{\partial}{\partial \bar{x}} \frac{\delta^{2} \bar{\Phi}}{\delta \bar{y}(\bar{x}) \delta \bar{y}(\bar{x})}+v \frac{\partial^{2}}{\partial \bar{x}^{2}} \frac{\delta \bar{\Phi}}{\delta \bar{y}(\bar{x})}\right) d \bar{x} & =\int y(x)\left(i \frac{\partial}{\partial x} \frac{\delta^{2} \Phi}{\delta y(x) \delta y(x)}+v \frac{\partial^{2}}{\partial x^{2}} \frac{\delta \Phi}{\delta y(x)}\right) d x \\
& +i \varepsilon\left[\frac{\delta^{2} \Phi}{\delta y(x) \delta y(x)}\right]_{x=-\infty}^{x=\infty}+v \varepsilon\left[\frac{\partial}{\partial x} \frac{\delta \Phi}{\delta y(x)}\right]_{x=-\infty}^{x=\infty}
\end{aligned}
$$

As we have shown in Discussion E.4 of Section 3, it is incorrect to assume or to impose that all functional derivatives of $\Phi$ vanish for $|x| \rightarrow \infty$, and hence, it would be incorrect to regard the transformation $T_{6}$ of Equation (A3) in general, i.e., for all possible realizations $\Phi$, as a symmetry of the Hopf-Burgers equation.

Note that besides the fact that the transformation $T_{6}$ of Equation (A3) is in general not admitted as a symmetry, the transformation itself is even unphysical. As was shown in $[16,18]$, this transformation violates the classical principle of cause and effect, and henceforth, the transformation 
$\mathrm{T}_{6}$ of Equation (A3) should be discarded altogether. The same is also true for the new statistical scaling symmetry $X_{7}^{\text {phys }}$ [1] (p. 1562). Although this scaling transformation (in contrast to $X_{6}^{\text {phys }}$ ) is admitted as a symmetry by the considered Hopf-Burgers Equation (A29), it nevertheless is unphysical in the same sense as $X_{6}^{\text {phys }}$ in that it also violates the classical causality principle and, hence, has to be discarded, as well; for more details, see $[19,20]$ and the review [21].

\section{References}

1. Janocha, D.D.; Wacławczyk, M.; Oberlack, M. Lie symmetry analysis of the Hopf functional-differential equation. Symmetry 2015, 7, 1536-1566.

2. Ibragimov, N.H. Lie Group Analysis of Differential Equations; CRC Press: Boca Raton, FL, USA, 1994; Volume I-III.

3. Ibragimov, N.H.; Kovalev, V.F.; Pustovalov, V.V. Symmetries of integro-differential equations: A survey of methods illustrated by the Benny equations. Nonlinear Dyn. 2002, 28, 135-153.

4. Fushchich, W.I.; Shtelen, W.M.; Serov, N.I. Symmetry Analyis and Exact Solutions of Equations of Nonlinear Mathematical Physics; Springer: Berlin, Germany, 1993.

5. Zawistowski, Z.J. Symmetries of integro-differential equations. Rep. Math. Phys. 2001, 48, 269-276.

6. Zawistowski, Z.J. General criterion of invariance for integro-differential equations. Rep. Math. Phys. 2004, 54, 251-260.

7. Olver, P.J. Applications of Lie Groups to Differential Equations, 2nd ed.; Springer: Berlin, Germany, 1993.

8. Bluman, G.W.; Cheviakov, A.F.; Anco, S.C. Applications of Symmetry Methods to Partial Differential Equations; Springer: Berlin, Germany, 2010.

9. Grigoriev, Y.N.; Ibragimov, N.H.; Kovalev, V.F.; Meleshko, S.V. Symmetries of Integro- Differential Equations: With Applications in Mechanics and Plasma Physics; Springer: Berlin, Germany, 2010.

10. Kevorkian, J.; Cole, J.D. Multiple Scale and Singular Perturbation Methods; Springer: Berlin, Germany, 1996.

11. Hosokawa, I.; Yamamoto, K. Numerical study of the Burgers' model of turbulence based on the characteristic functional formalism. Phys. Fluids 1970, 13, 1683-1692.

12. Kannan, R.; Wang, Z.J. A high order spectral volume solution to the Burgers' equation using the Hopf-Cole transformation. Int. J. Numer. Methods Fluids 2012, 69, 781-801.

13. Hopf, E. Statistical hydromechanics and functional calculus. J. Rat. Mech. Anal. 1952, 1, 87-123.

14. Oberlack, M.; Wacławczyk, M. On the extension of Lie group analysis to functional differential equations. Arch. Mech. 2006, 58, 597-618.

15. Wacławczyk, M.; Oberlack, M. Application of the extended Lie group analysis to the Hopf functional formulation of the Burgers equation. J. Math. Phys. 2013, 54, 072901.

16. Frewer, M.; Khujadze, G.; Foysi, H. Comment on "Application of the extended Lie group analysis to the Hopf functional formulation of the Burgers equation". J. Math. Phys. 2016, 57, 034102.

17. Klauder, J.R. A Modern Approach to Functional Integration; Springer: Berlin, Germany, 2011.

18. Frewer, M.; Khujadze, G.; Foysi, H. Comment on "Statistical symmetries of the Lundgren-Monin-Novikov hierarchy". Phys. Rev. E 2015, 92, 067001.

19. Frewer, M.; Khujadze, G.; Foysi, H. On the physical inconsistency of a new statistical scaling symmetry in incompressible Navier-Stokes turbulence. 2014, arXiv:1412.3061.

20. Frewer, M.; Khujadze, G.; Foysi, H. A critical examination of the statistical symmetries admitted by the Lundgren-Monin-Novikov hierarchy of unconfined turbulence. 2014, arXiv:1412.6949.

21. Frewer, M.; Khujadze, G.; Foysi, H. A note on the notion "statistical symmetry". 2016, arXiv:1602.08039.

(C) 2016 by the authors; licensee MDPI, Basel, Switzerland. This article is an open access article distributed under the terms and conditions of the Creative Commons Attribution (CC-BY) license (http://creativecommons.org/licenses/by/4.0/). 